\title{
Electroweak vacuum lifetime in two Higgs doublet models
}

\author{
V. Branchina, ${ }^{a, b}$ F. Contino ${ }^{a, b, c}$ and P.M. Ferreira ${ }^{d, e}$ \\ ${ }^{a}$ Department of Physics and Astronomy, University of Catania, \\ Via Santa Sofia 64, 95123 Catania, Italy \\ ${ }^{b}$ INFN, Sezione di Catania, \\ Via Santa Sofia 64, 95123 Catania, Italy \\ ${ }^{c}$ Scuola Superiore di Catania, \\ Via Valdisavoia 9, 95123 Catania, Italy \\ ${ }^{d}$ Instituto Superior de Engenharia de Lisboa - ISEL, \\ 1959-007 Lisboa, Portugal \\ ${ }^{e}$ Centro de Física Teórica e Computacional, Faculdade de Ciências, Universidade de Lisboa, \\ Av. Prof. Gama Pinto 2, 1649-003 Lisboa, Portugal \\ E-mail: vincenzo.branchina@ct.infn.it, filippo.contino@ct.infn.it, \\ pmmferreira@fc.ul.pt
}

ABSTRACT: We study the stability of neutral electroweak vacua in two Higgs doublet models, and calculate the lifetime of these states when the parameters are such that they are false vacua. As the two Higgs doublet model is invariant under a sign change of both doublets, degenerate true vacua exist. It is shown that this degeneracy, despite the fact that each of these minima locally describes the same physics, can immensely affect their lifetime. We apply these calculations to the parameter space of the models which is allowed by recent LHC searches, and infer combinations of parameters which should be excluded on grounds of a tunneling time inferior to the age of the universe.

Keywords: Beyond Standard Model, Higgs Physics

ArXiv ePrint: 1807.10802 


\section{Contents}

1 Introduction 1

2 The two-Higgs doublet model potential 4

2.1 Theoretical constraints on quartic couplings 6

$\begin{array}{lll}2.2 & \text { The electroweak-breaking minimum } & 7\end{array}$

2.3 Experimental constraints on the $2 \mathrm{HDM} \quad 8$

3 Coexisting minima in the 2HDM 10

4 Tunneling and bounces 13

5 Tunneling to degenerate vacua $\quad 18$

$\begin{array}{lll}6 & 2 H D M & 22\end{array}$

6.1 General scans for type I and II models 23

6.2 First benchmark scenarios: safe regions 24

6.3 Second benchmark scenario: considerable exclusion 27

$\begin{array}{llr}7 & \text { Conclusions } & 28\end{array}$

\section{Introduction}

In 2012 the LHC discovered the last missing piece of the Standard Model (SM) of particle physics $[1,2]$, the Higgs boson. We are now measuring with increasing precision the properties of this new particle, thus probing the hitherto unknown scalar sector of the theory. The trend in the ATLAS and CMS results is clear: the $125 \mathrm{GeV}$ scalar discovered behaves very much like the SM Higgs boson was expected to behave (see, for instance, [3]). However, the current experimental results for the scalar sector still leave a lot of room for Beyond Standard Model (BSM) physics to occur, and BSM is required to explain a great number of phenomena which the SM cannot account for, of which the origin of dark matter, dark energy and the matter-antimatter asymmetry are but three amongst many other issues to be resolved.

The two-Higgs doublet model (2HDM) $[4,5]$ is arguably the simplest SM extension, in which the particle content of the SM is complemented by a second Higgs doublet. The model boasts a rich phenomenology, with a larger scalar sector, including two CP-even scalars, a pseudoscalar and a charged scalar, and may have spontaneous CP breaking for certain choices of its parameters, thus offering an additional source of $\mathrm{CP}$ violation which might help in explaining why the universe contains so much more matter than antimatter. In one version of the 2HDM - the so called inert model [6-9] - dark matter candidates arise naturally, their lack of interactions with "normal" matter being ensured by a discrete symmetry unbroken by the vacuum. The $2 \mathrm{HDM}$ does an excellent job at fitting the existing experimental data. The properties of the discovered scalar, very similar to those expected within the SM, can easily be reproduced in the $2 \mathrm{HDM}$, as seen soon 
after discovery $[10,11]$ and is today easily verified for much of the parameter space using, for instance, the HiggsSignals code [12]. Of course, the 2HDM predicts the existence of other scalars, as yet not discovered, so the model must also be in agreement with current experimental searches for BSM particles. Even after demanding that the $125 \mathrm{GeV}$ scalar be SM-like, there remains a large $2 \mathrm{HDM}$ parameter space available to comply with those experimental results (as proven by application of the HiggsBounds code [13-15]). Also, the model can easily fit constraints on the charged Higgs mass arising from other observables, such as B-meson physics [16-22].

Another interesting property of the $2 \mathrm{HDM}$ is that already at the classical level it has a richer vacuum structure - whereas in the classical SM potential there can only be one type of minimum, the $2 \mathrm{HDM}$ has the possibility of three physically different kinds of minima: an electroweak-breaking but CP-and-charge preserving (we call it "normal" minimum), analogous to the SM; a minimum which spontaneously breaks both the electroweak and $\mathrm{CP}$ symmetries; and a minimum where the vacuum expectation value (vev) of the scalar doublets carries electric charge, and electric charge conservation no longer holds. However, the scalar potential of the model is such that, at least at tree level, minima of a different nature cannot simultaneously coexist [23-26]. In other words, if for instance a normal minimum exists, which is nothing more than an electroweak (EW) minimum which preserves charge and $\mathrm{CP}$, any possible charge breaking $(\mathrm{CB})$ or $\mathrm{CP}$ breaking stationary points of the potential are necessarily saddle points, and in addition lie above the EW minimum. Similar conclusions are valid for CB or CP minima - if they exist, all other types of possible stationary points will be saddle points lying above them. The stability of a $2 \mathrm{HDM}$ vacuum against tunneling to another vacuum of a different nature is therefore ensured by the theory itself, at least at tree level.

There is however another $2 \mathrm{HDM}$ property concerning normal vacua: for certain regions of the parameter space, there may exist two non-degenerate vacua of this type [25-27], both of them $\mathrm{CP}$ and charge preserving, but having vevs which break the electroweak symmetry. However, the value of those vevs is different for each minimum, which means that, since all elementary particles gain their mass from interactions with scalar particles, the mass spectrum at each of the two minima is quite different. In other words, the vevs of the doublets, $v_{i}$, are such that in "our" minimum they satisfy $v_{1}^{2}+v_{2}^{2}=246 \mathrm{GeV}^{2}$ - thus in "our" minimum all elementary particles have their well-known masses - whereas a different mass spectrum holds for the second minimum. If the EW vacuum which the universe currently occupies is not the absolute minimum of the potential, it will sooner or later tunnel to a deeper minimum that breaks the same symmetries. The (surprisingly simple) conditions under which a second $2 \mathrm{HDM}$ minimum may exist, and the condition which discriminates whether "our" minimum is the global one were established in refs. [25, 26, 28, 29]. The deeper vacuum, different from the "standard" EW breaking one, was dubbed panic vacuum in [28, 29]: a transition from the EW minimum to the deeper one would be disastrous. In fact, such a transition would release a colossal amount of energy; and since the fields in the two minima have different vevs, all elementary particles would change their masses upon transition to the deeper vacuum. Both situations are nothing short of catastrophic. 
The mere existence of a deeper, "panic" vacuum, however, is not sufficient to exclude the parameters of the potential which yield such a bizarre possibility. In fact, if the tunneling time $\tau$ from the false to the true vacuum is larger than the age of the universe, the existence of the deeper vacuum would have no impact whatsoever in the phenomenology observed while the universe lies in the upper minimum. Thus the computation of $\tau$ becomes a fundamental tool to distinguish between those regions of the parameter space which yield dangerous panic vacua, and those for which the deeper vacua exist but are practically harmless.

It is now worth it to stress an important difference between the 2HDM and the SM. In the latter case the EW vacuum shows its instability (metastability) only once radiative corrections are taken into account, and this is mainly due to the negative contribution to the potential coming from the top quark. Actually the Higgs effective potential $V(\phi)$ turns over for values of $\phi>v=246 \mathrm{GeV}$, and for the present experimental values of $m_{h}$ and $m_{t}$, namely $m_{h} \sim 125.09 \mathrm{GeV}$ and $m_{t} \sim 173.34 \mathrm{GeV} \quad[30,31]$, it develops a second minimum much deeper than the EW one and at a much larger value of the field. Under these conditions, then, the EW minimum is a metastable state, a so called "false vacuum" $\left(\phi_{\mathrm{fv}} \equiv v\right)$, while the deeper minimum is the true vacuum (that occurs at $\left.\phi_{\mathrm{tv}} \gg v\right)$. Unlike the SM case, however, the coexistence of two minima in the $2 \mathrm{HDM}$ potential already occurs at tree-level, and the analysis of the stability of the false vacuum (the EW minimum in our case) can be already undertaken at this level (i.e. prior to the study of the loop corrected potential), as was the case for the pioneering work of Coleman and collaborators [32, 33], where a (classical) potential with two minima of different depths was considered.

Physically the false vacuum decay is triggered by quantum fluctuations, that induce a finite probability for a bubble of true vacuum to materialize in a false vacuum sea. Both in flat and curved spacetime backgrounds, Coleman and collaborators considered a scalar theory where the potential $V(\phi)$ has a relative and an absolute minimum, at $\phi_{\mathrm{fv}}$ and $\phi_{\mathrm{tv}}$ respectively, such that the energy density difference $V\left(\phi_{\mathrm{fv}}\right)-V\left(\phi_{\mathrm{tv}}\right)$ is much smaller than the height of the "potential barrier" $V\left(\phi_{\mathrm{top}}\right)-V\left(\phi_{\mathrm{fv}}\right)$, where $V\left(\phi_{\mathrm{top}}\right)$ is the maximum of the potential between the two minima. Under these conditions the true vacuum bubble is separated from the false vacuum sea by a "thin wall", and this allows us to treat the problem analytically, within the so called "thin wall" approximation.

The conditions under which this approximation can be applied are however not fulfilled in the SM case, at least for the central values of the Higgs and top masses reported above, but this is not a problem because the stability analysis can be performed numerically. If the EW vacuum is metastable, the fate of our universe is to decay sooner or later into the true vacuum, and it is then of the greatest importance to estimate its lifetime $\tau$. When the SM alone is considered, the masses of the Higgs boson and of the top quark are taken as reported above, and it is also assumed that interactions at higher energy scales have no impact on the stability condition of the EW vacuum, $\tau$ turns out to be much larger than the age of the universe $T_{U}[34,35]$. Actually $\tau \sim 10^{640} T_{U}\left(T_{U} \sim 13,7 \cdot 10^{9}\right.$ years $)$, and accordingly we can say that our universe is practically stable.

It was later realized, however, that the stability condition of the EW vacuum is very sensitive to unknown New Physics (even if that physics occurs at high energy scales), 
and the decay rate of the EW vacuum can be strongly modified by its presence [36-41]. This leads to the conclusion that models of BSM physics should not only satisfy all current experimental constraints stemming from precision measurements, but should also be tested against a careful stability analysis, as only models for which the EW vacuum lifetime turns out to be larger than the age of the universe are physically acceptable.

In this paper, we will undertake a thorough analysis of the tunneling between neutral vacua in the $2 \mathrm{HDM}$, by calculating the tunneling time from false to true vacua. To this end, we have to look for the so-called bounce solutions to the Euclidean Euler-Lagrange equations that have $\mathrm{O}(4)$ symmetry and satisfy specific boundary conditions [32]. In principle these bounces are composed by eight fields, since the two doublets have eight real components, unlike the SM case where only one field is present. Using gauge invariance arguments, however, we will show that the problem is reduced to a five-field calculation. Analysing the bounce equations, from which tunneling times are computed, we will show that for CP-conserving potentials the problem is further reduced to a two-field calculation - but we will also show that for potentials with explicit CP violation this is no longer the case, and in general the bounce solutions will involve three fields. Further consideration of the full minimum landscape of the $2 \mathrm{HDM}$ leads to the conclusion that the lifetime of false vacua may be enormously affected by the existence of degenerate true vacua, which is caused by the invariance of the potential under a sign swap of both doublets. Using a dedicated and very efficient code to compute tunneling times in theories with many fields [42], we will probe large regions of $2 \mathrm{HDM}$ parameter space - complying with all theoretical and experimental constraints that the model is expected to obey in this LHC era - and verify under which conditions dangerous deeper minima develop. We will show that specific benchmarks of the model - for which we specify 6 out of 8 of the scalar sector parameters - may be completely safe, boasting either a single minimum or tunneling times to deeper minima far larger than the age of the universe. Nonetheless, other perfectly banal benchmarks may have over $11 \%$ of the remaining 2-parameter space excluded on account of having far too short tunneling times to a deeper vacuum.

\section{The two-Higgs doublet model potential}

The 2HDM is perhaps the simplest extension of the SM - the particle content of the 2HDM is enlarged by a second $\mathrm{SU}(2)_{W} \times \mathrm{U}(1)_{Y}$ doublet, but the gauge and fermion content of the model is the same as the SM's. The model was proposed by T.D. Lee in 1973 [4] as a means to obtain CP violation from spontaneous symmetry breaking. For a review, see [5]. The model therefore contains two hypercharge 1 doublets, $\Phi_{1}$ and $\Phi_{2}$, in terms of which the most general renormalizable $2 \mathrm{HDM}$ scalar potential is written as

$$
\begin{aligned}
V= & m_{11}^{2}\left|\Phi_{1}\right|^{2}+m_{22}^{2}\left|\Phi_{2}\right|^{2}-\left(m_{12}^{2} \Phi_{1}^{\dagger} \Phi_{2}+\text { h.c. }\right) \\
& +\frac{1}{2} \lambda_{1}\left|\Phi_{1}\right|^{4}+\frac{1}{2} \lambda_{2}\left|\Phi_{2}\right|^{4}+\lambda_{3}\left|\Phi_{1}\right|^{2}\left|\Phi_{2}\right|^{2}+\lambda_{4}\left|\Phi_{1}^{\dagger} \Phi_{2}\right|^{2} \\
& +\left[\frac{1}{2} \lambda_{5}\left(\Phi_{1}^{\dagger} \Phi_{2}\right)^{2}+\lambda_{6}\left|\Phi_{1}\right|^{2}\left(\Phi_{1}^{\dagger} \Phi_{2}\right)+\lambda_{7}\left|\Phi_{2}\right|^{2}\left(\Phi_{1}^{\dagger} \Phi_{2}\right)+\text { h.c. }\right],
\end{aligned}
$$


where the coefficients $m_{12}^{2}, \lambda_{5,6,7}$ can be complex. The doublets $\Phi_{1}$ and $\Phi_{2}$ are not physical fields - the mass eigenstates which arise from them are physical, but the doublets themselves are not. This means that any linear combination of the doublets which preserves the form of the model's kinetic terms provides an equally valid physical description of physics - this corresponds to an invariance of the model under fields redefinitions, so called basis changes of the form $\Phi_{i}^{\prime}=U_{i j} \Phi_{j}$, where $U$ is a $2 \times 2$ unitary matrix. Though the potential of eq. (2.1) seemingly has 14 independent real parameters, the freedom to redefine the doublets means that in fact one can eliminate three of those parameters, and thus the most general 2HDM scalar potential has 11 independent real parameters [43].

When considering the whole theory we must include scalar-fermion interactions - the Yukawa sector. And there we run into a problem - if we build the most general lagrangian with two Higgs doublets, the Yukawa sector will include tree-level flavour changing neutral currents (FCNC) mediated by neutral scalars. This happens because the most general Yukawa terms of the 2HDM include interactions of both doublets with all fermions. However, these FCNC are very tightly constrained by experimental data and they should be avoided. This may be achieved, of course, by simply fine tuning the Yukawa couplings - there is sufficient freedom in the Yukawa coupling matrices to achieve this. One other possibility is to assume an "alignment" ansatz relating Yukawa matrices [44-48]. The most studied model, however, eliminates tree-level scalar-mediated FCNC by imposing a $\mathbb{Z}_{2}$ discrete symmetry upon the model - this method, unlike the previous ones, is entirely stable under renormalization. The discrete symmetry usually considered demands that the lagrangian be invariant under a transformation on the doublets of the form $\Phi_{1} \rightarrow \Phi_{1}$ and $\Phi_{2} \rightarrow-\Phi_{2}[49,50]$. As a consequence, the parameters $m_{12}^{2}, \lambda_{6}$ and $\lambda_{7}$ vanish from the potential - though $m_{12}^{2}$ is reintroduced as a (real) soft-breaking term, to enlarge the allowed parameter space and, among other things, allow the theory to have a decoupling limit [43] where the masses of all scalars other than the SM-like one can be made very large. The final potential with which we will be working is thus

$$
\begin{aligned}
V= & m_{11}^{2}\left|\Phi_{1}\right|^{2}+m_{22}^{2}\left|\Phi_{2}\right|^{2}-m_{12}^{2}\left(\Phi_{1}^{\dagger} \Phi_{2}+\text { h.c. }\right) \\
& +\frac{1}{2} \lambda_{1}\left|\Phi_{1}\right|^{4}+\frac{1}{2} \lambda_{2}\left|\Phi_{2}\right|^{4}+\lambda_{3}\left|\Phi_{1}\right|^{2}\left|\Phi_{2}\right|^{2}+\lambda_{4}\left|\Phi_{1}^{\dagger} \Phi_{2}\right|^{2}+\frac{1}{2} \lambda_{5}\left[\left(\Phi_{1}^{\dagger} \Phi_{2}\right)^{2}+\text { h.c. }\right],
\end{aligned}
$$

where now all parameters are real (we have further imposed CP conservation on the potential, which makes all possible complex phases vanish).

The 2HDM, of course, is not only a theory of the scalar sector, it includes also gauge bosons and three generations of fermions, as does the SM. The most general Yukawa sector of the model, as mentioned above, will generate tree-level FCNC which are strongly disfavoured by experimental results. These are eliminated imposing, on the full lagrangian, the discrete symmetry $\Phi_{1} \rightarrow \Phi_{1}$ and $\Phi_{2} \rightarrow-\Phi_{2}$ and we have already explained the impact of this symmetry on the scalar sector; on the Yukawa sector, it forces only one of the doublets to couple (and thus give mass) to each generation of like-charged fermions. Depending on how the fermionic fields (both the left doublets and right singlets) transform under the $\mathbb{Z}_{2}$ symmetry, there are then several possible types of $2 \mathrm{HDM}$, with different 
phenomenologies and classified according to their scalar-fermion interactions. Usually, one considers four different types: ${ }^{1}$

- Model Type I, where all fermions couple to a single Higgs doublet, chosen as $\Phi_{2}$ per convention.

- Model Type II, where all right-handed up-type quarks couple to $\Phi_{2}$, but right-handed down-type quarks and charged leptons couple to $\Phi_{1}$. This type of couplings is analogous to what happens in SUSY models.

- The Lepton-specific model, in which all quarks couple to $\Phi_{2}$, but right-handed charged leptons couple to $\Phi_{1}$.

- The Flipped model, in which right handed up quarks and charged leptons couple to $\Phi_{2}$, but right-handed down quarks couple to $\Phi_{1}$.

Thus for each model each same-charge type of fermions may gain their masses from different Higgs doublets. The fact that only one Higgs doublet couples to fermions of the same electric charge eliminates tree-level FCNC, as the couplings between the physical scalar particles and the fermions will be described by diagonal matrices [5]. As already mentioned, each of these models has different phenomenologies, a subject we will address in section 2.3.

\subsection{Theoretical constraints on quartic couplings}

Notice that the quartic couplings of (2.2) are not completely unconstrained - in order to ensure that the potential is bounded from below (BFB), meaning, no directions in field space along which the potential can tend to minus-infinity, the couplings need to obey [6]

$$
\begin{array}{rlrl}
\lambda_{1} & >0, & \lambda_{2} & >0, \\
\lambda_{3}>-\sqrt{\lambda_{1} \lambda_{2}}, & \lambda_{3}+\lambda_{4}-\left|\lambda_{5}\right| & >-\sqrt{\lambda_{1} \lambda_{2}} .
\end{array}
$$

It has been proven that these (tree-level) conditions are both necessary and sufficient [25, 26]. It is possible to go beyond tree-level in these BFB constraints - this is usually accomplished by studying the renormalization group evolution of the quartic couplings of the potential and imposing that the conditions shown in eqs. (2.3) be valid at all scales. Prior to the discovery of the Higgs boson, this procedure was used to constrain the 2HDM parameter space (see, for instance, [51-54]). Post-Higgs discovery, this method has shown that the metastability claimed for the SM effective potential $[35,55,56]$, which seemingly may develop a deeper minimum if the theory is considered valid all the way up to the Planck scale, may be cured in the 2HDM due to its larger scalar content [48, 57-64]. In the current work we will confine ourselves to tree-level conditions. ${ }^{2}$ Another set of constraints upon the potential's parameters arises from requiring that the theory be unitary - this

\footnotetext{
${ }^{1}$ The number of possible models would increase if one were to consider also the possible interaction terms between the scalar doublets and neutrinos, which we will not do in the current work.

${ }^{2}$ In any case, since we will only consider regions of the 2 HDM well within the so-called "alignment limit", we expect that the tree-level conditions will be more than sufficient for the model to be valid up to very high scales [64].
} 
translates into further constraints upon the quartic couplings of the potential, which may be reduced to [65-67]

$$
\begin{aligned}
\left|\lambda_{3}-\lambda_{4}\right| & <8 \pi \\
\left|\lambda_{3}+2 \lambda_{4} \pm 3 \lambda_{5}\right| & <8 \pi \\
\mid \frac{1}{2}\left(\lambda_{1}+\lambda_{2}+\sqrt{\left(\lambda_{1}-\lambda_{2}\right)^{2}+4 \lambda_{4}^{2}}\right) & <8 \pi \\
\mid \frac{1}{2}\left(\lambda_{1}+\lambda_{2}+\sqrt{\left(\lambda_{1}-\lambda_{2}\right)^{2}+4 \lambda_{5}^{2}}\right) & <8 \pi .
\end{aligned}
$$

Again, we will consider tree-level unitarity constraints, though one-loop contributions have been considered, and shown to curtail the available 2HDM parameter space [62, 68].

\subsection{The electroweak-breaking minimum}

The potential described by eq. (2.2) can yield, depending of the values of the parameters, different types of minima. The scalar fields can acquire vacuum expectation values (vevs) and break the symmetries of the model in different ways. We call "normal vacuum" the case where both doublets acquire real and neutral vevs,

$$
\left\langle\Phi_{1}\right\rangle_{N}=\frac{1}{\sqrt{2}}\left(\begin{array}{c}
0 \\
v_{1}
\end{array}\right),\left\langle\Phi_{2}\right\rangle_{N}=\frac{1}{\sqrt{2}}\left(\begin{array}{c}
0 \\
v_{2}
\end{array}\right) .
$$

These normal minima are similar to the SM vacuum - they break the same gauge symmetries and preserve $\mathrm{CP}$, and constitute the focus of the work of this paper (we will briefly discuss other types of possible $2 \mathrm{HDM}$ minima in section 3 ). Let us now define the (real) components of the doublets $\Phi_{1}$ and $\Phi_{2}$ as

$$
\Phi_{1}=\frac{1}{\sqrt{2}}\left(\begin{array}{c}
\varphi_{c 1}+\mathrm{i} \varphi_{c 2} \\
\varphi_{r 1}+\mathrm{i} \varphi_{i 1}
\end{array}\right), \quad \Phi_{2}=\frac{1}{\sqrt{2}}\left(\begin{array}{c}
\varphi_{c 3}+\mathrm{i} \varphi_{c 4} \\
\varphi_{r 2}+\mathrm{i} \varphi_{i 2}
\end{array}\right),
$$

where the upper components correspond to charged $(+1)$ fields and the lower components, to neutral ones. When the potential develops a normal minimum, the real neutral components, $\varphi_{r 1}$ and $\varphi_{r 2}$, give rise to two mass eigenstates which correspond to CP-even scalars, dubbed $h$ and $H$. On the other hand, the imaginary components, $\varphi_{i 1}$ and $\varphi_{i 2}$, originate a pseudoscalar particle, $A$, and the neutral Goldstone boson $G^{0}$ which provides the $Z$ boson with its mass. Finally, the upper, charged components $\varphi_{c i}$ yield a charged Higgs scalar, $H^{ \pm}$and the charged Goldstone boson $G^{ \pm}$which gives mass to the $W$ gauge bosons. For such normal minima it is also customary to define two angles: the ratio of the vevs $v_{1}$ and $v_{2}$ defines the angle $\beta$, such that

$$
\tan \beta=\frac{v_{2}}{v_{1}} .
$$

$\beta$ is the angle which diagonalizes both the charged and pseudoscalar squared scalar mass matrices, and can be considered to only take values between 0 and $\pi / 2$ without loss of generality. ${ }^{3}$ On the other hand, the CP-even squared scalar mass matrix is diagonalized

\footnotetext{
${ }^{3}$ This choice is valid for one specific vacuum, other vacua may have vevs of different signs.
} 
by a different angle, $\alpha$, defined such that the two physical eigenstates, $h$ and $H$, are related to the neutral real components of the doublets as

$$
\begin{aligned}
h & =\sin \alpha \varphi_{r 1}-\cos \alpha \varphi_{r 2} \\
H & =-\cos \alpha \varphi_{r 1}-\sin \alpha \varphi_{r 2} .
\end{aligned}
$$

Again without loss of generality, this angle can be chosen such that $-\pi / 2 \leq \alpha \leq \pi / 2$. The minimization conditions relate the vevs of eq. (2.5) to the parameters of the potential, such that

$$
\begin{aligned}
& m_{11}^{2} v_{1}-m_{12}^{2} v_{2}+\frac{\lambda_{1}}{2} v_{1}^{3}+\frac{\lambda_{345}}{2} v_{2}^{2} v_{1}=0 \\
& m_{22}^{2} v_{2}-m_{12}^{2} v_{1}+\frac{\lambda_{2}}{2} v_{2}^{3}+\frac{\lambda_{345}}{2} v_{1}^{2} v_{2}=0
\end{aligned}
$$

where we have defined

$$
\lambda_{345} \equiv \lambda_{3}+\lambda_{4}+\lambda_{5}
$$

Notice that, since the potential is invariant under a sign change for both doublets, if eqs. (2.9) admit a solution $\left\{v_{1}, v_{2}\right\}$ obviously $\left\{-v_{1},-v_{2}\right\}$ will also be a solution. Also obviously, this second solution will be physically indistinguishable from the first one. This seemingly trivial point will be extremely important later on, and we will show in section 5 that it can have a stunning impact on the tunneling rates between vacua.

Instead of the potential's couplings, we can choose to describe the model in terms of the four physical masses, $m_{h}=125 \mathrm{GeV}, m_{H}, m_{A}$ and $m_{H^{ \pm}}$, the angles $\beta$ and $\alpha$, the vev $v=246 \mathrm{GeV}$ and a further parameter, for instance the soft breaking term $m_{12}^{2}-$ a total of eight parameters, just as the potential of eq. (2.2). The quartic couplings of the model can then be expressed as

$$
\begin{aligned}
& \lambda_{1}=\frac{1}{v^{2} c_{\beta}^{2}}\left(c_{\alpha}^{2} m_{H}^{2}+s_{\alpha}^{2} m_{h}^{2}-m_{12}^{2} \frac{s_{\beta}}{c_{\beta}}\right), \\
& \lambda_{2}=\frac{1}{v^{2} s_{\beta}^{2}}\left(s_{\alpha}^{2} m_{H}^{2}+c_{\alpha}^{2} m_{h}^{2}-m_{12}^{2} \frac{c_{\beta}}{s_{\beta}}\right), \\
& \lambda_{3}=\frac{1}{v^{2}}\left[2 m_{H^{ \pm}}^{2}+\frac{s_{2 \alpha}\left(m_{H}^{2}-m_{h}^{2}\right)}{s_{2 \beta}}-\frac{m_{12}^{2}}{s_{\beta} c_{\beta}}\right], \\
& \lambda_{4}=\frac{1}{v^{2}}\left(m_{A}^{2}-2 m_{H^{ \pm}}^{2}+\frac{m_{12}^{2}}{s_{\beta} c_{\beta}}\right), \\
& \lambda_{5}=\frac{1}{v^{2}}\left(\frac{m_{12}^{2}}{s_{\beta} c_{\beta}}-m_{A}^{2}\right),
\end{aligned}
$$

where for simplification we defined $s_{\theta}=\sin \theta$ and $c_{\theta}=\cos \theta$.

\subsection{Experimental constraints on the 2HDM}

The larger scalar content of the 2HDM, compared with the SM, leads to measurable impacts on several experimental observables. So far no scalars other than the $125 \mathrm{GeV}$ one have been discovered - and therefore BSM searches at the LHC and elsewhere impose bounds on the 
masses and couplings of the extra scalars of the 2HDM. Further, even before the discovery of the Higgs boson, electroweak precision studies from LEP and other accelerators were used to curtail the values of BSM models, including the $2 \mathrm{HDM}$. A charged scalar such as the one predicted by the $2 \mathrm{HDM}$ has considerable contributions to several B-meson observables, and data from B-physics measurements constitute some of the model's most stringent constraints. In the current work we incorporated a wealth of experimental constraints in the parameter scans used in section 6 .

In general, BSM physics may have substantial contributions to Electroweak Precision Constraints (EWPC), namely the oblique $S, T$ and $U$ parameters [69-71]. These constraints may, for instance, force the charged Higgs mass and the pseudoscalar one to be very close in value. We computed these oblique parameters and used the most recent fit [72] to constrain the $2 \mathrm{HDM}$ parameter space. Direct searches from LEP, using the channel $e^{+} e^{-} \rightarrow H^{+} H^{-}$[73], impose a lower bound on the charged Higgs mass of roughly $80 \mathrm{GeV}$, which we also implemented [21]. And, as described above, the 2HDM contributions to B-physics observables, such as the values of the $b \rightarrow s \gamma$ decay rate [16-20] and the $Z \rightarrow b \bar{b}$ width [16, 22], impose considerable constraints, usually expressed as exclusions on the $m_{H^{ \pm}}-\tan \beta$ plane. Roughly speaking, these constraints translate as requiring that $\tan \beta$ be above 1 for most of the parameter space in all model types, and an almost $\tan \beta$ independent lower bound on the charged Higgs mass for model type II (and Flipped), of roughly $\sim 580 \mathrm{GeV}[20]$. Other flavour constraints, such as those arising from $B \rightarrow \tau \nu$, $\Delta M_{B_{s, d}}$, etc. [21], were also taken into account.

The Higgs boson discovery at the LHC has been followed by many measurements of this particle's properties, which have been seen to be very much in agreement with what one could expect for a SM-like scalar. The experimental results are thus pushing the 2HDM into the so-called "alignment limit", wherein the $125 \mathrm{GeV}$ state is almost "aligned" with one of the doublets (this in practice corresponds to values of $\sin (\beta-\alpha)$ very close to 1 ), and the remaining scalars sufficient massive, or with sufficiently weak interactions, to have eluded detection thus far. In practical terms, the LHC constraints are obtained from the $\mu$ ratios between the observed number of events in some Higgs-mediated channel, and the SM expected value for the same quantity. For the 2HDM, then, the quantities to compare with experimental results such as those from [3] are

$$
\mu_{X}=\frac{\sigma^{2 \mathrm{HDM}}(p p \rightarrow h)}{\sigma^{\mathrm{SM}}(p p \rightarrow h)} \frac{B R^{2 \mathrm{HDM}}(h \rightarrow X)}{B R^{\mathrm{SM}}(h \rightarrow X)},
$$

where $\sigma$ stands for the production cross section of $h$ in proton-proton collisions at the LHC and $B R$ for the decay branching ratios of $h$ to some final state $X$, such as $Z Z, W W$, $\gamma \gamma, b \bar{b}, \ldots$ The fact that $h$ is behaving in a SM-like manner means that the measured values for these $\mu_{X}$ are close to one, but the current experimental uncertainties still allow values with deviations larger than $30 \%$ from unity. In our calculations we will consider mostly scalars produced via the main channel of gluon-gluon fusion, the cross sections of such processes being obtained by SusHiv1.6.0 [74, 75], at NNLO QCD. Other production channels (such as VBF, $b \bar{b} h$ or $t \bar{t} h$ ) were also computed, but since they are subdominant, for the purposes of the current paper we chose not to use them. As for the branching 
ratios, all decay widths were computed at leading order, with the necessary NLO QCD corrections to the $b \bar{b}$ width taken into account. In fact, requiring that $\mu_{Z Z}, \mu_{\gamma \gamma}, \mu_{b \bar{b}}$ and $\mu_{\tau \bar{\tau}}$ be within $30 \%$ of their SM value (i.e., all $\mu$ 's having values in the interval 0.7 to 1.3 ) is enough to have a rough compliance with the $2 \times 1 \sigma$ experimental precision from [3].

Finally, there is a wealth of results on searches for the extra scalars predicted in the 2HDM (see ref. [76] and references therein, for a review of the status of the diverse search channels), with measurements imposing exclusion regions in the parameter space of the model. By and large, requiring that the $125 \mathrm{GeV}$ state $h$ be very SM-like is sufficient to comply with most exclusion bounds for other scalar searches, even though there are exceptions [77], like pseudoscalar production and decay to $Z h$ in the wrong sign limit in the 2HDM [78-84]. For the purposes of the current work, in which we wish to show the possible importance of the tunneling time calculations in $2 \mathrm{HDM}$ parameter space, we have verified that in regions of parameters analysed the $30 \%$ bound on the several $\mu_{X}$ was sufficient to comply with extra scalar search results.

\section{Coexisting minima in the $2 \mathrm{HDM}$}

Since the 2HDM has a scalar potential much more elaborate than the SM one, it possesses therefore a richer vacuum structure. In fact, in the $2 \mathrm{HDM}$ three classes of vacua may occur, depending on the parameters of the model. The first corresponds to normal vacua, wherein the doublets have vevs such as those described by eq. (2.5). This kind of vacuum therefore breaks $\mathrm{SU}(2)_{L} \times \mathrm{U}(1)_{Y}$ down to $\mathrm{U}(1)_{e m}$, just as the $\mathrm{EW}$ vacuum in the $\mathrm{SM}$, therefore preserving both $\mathrm{CP}$ and the electromagnetic symmetry.

But vacua with a spontaneous breaking of $\mathrm{CP}$ are also possible, and in fact their existence is the main reason the model was created by T.D. Lee [4]. Such vacua occur when the doublets have neutral vevs, but now, unlike eq. (2.5), a relative complex phase between them appears, i.e. the vevs are of the form

$$
\left\langle\Phi_{1}\right\rangle_{\mathrm{CP}}=\frac{1}{\sqrt{2}}\left(\begin{array}{c}
0 \\
\bar{v}_{1}
\end{array}\right), \quad\left\langle\Phi_{2}\right\rangle_{\mathrm{CP}}=\frac{1}{\sqrt{2}}\left(\begin{array}{c}
0 \\
\bar{v}_{2} \exp ^{i \theta}
\end{array}\right),
$$

with $\theta \neq n \pi$, for any integer $n$. The complex phase induces spontaneous CP breaking and the resulting scalar mass eigenstates have no definite $\mathrm{CP}$ quantum numbers - they are neither CP-even nor CP-odd. As a consequence, the neutral mass matrix in such minima is more complex than the analogous matrix in normal vacua: in the latter, a $4 \times 4$ matrix breaks into two $2 \times 2$ blocks, one having two non-zero eigenvalues, corresponding to the masses of the CP-even states $h$ and $H$, the other having a zero eigenvalue (the Goldstone boson $G^{0}$ ) and the pseudoscalar mass of $A$; in the former case, the $4 \times 4$ matrix does not reduce to two blocks, it possesses a zero eigenvalue (again the neutral Goldstone) and three eigenstates with interactions such that they are neither scalars nor pseudoscalars.

Charge breaking vacua are also a possibility, where the upper components of the doublets also acquire vevs, i.e. we will have

$$
\left\langle\Phi_{1}\right\rangle_{\mathrm{CB}}=\frac{1}{\sqrt{2}}\left(\begin{array}{c}
0 \\
v_{1}^{\prime}
\end{array}\right), \quad\left\langle\Phi_{2}\right\rangle_{\mathrm{CB}}=\frac{1}{\sqrt{2}}\left(\begin{array}{c}
v_{3}^{\prime} \\
v_{2}^{\prime}
\end{array}\right) .
$$


These minima, of course, are to be avoided at all costs - the charged vev $v_{3}^{\prime}$ above will break the electromagnetic symmetry and give the photon a mass. In the scalar mass matrix, the neutral (lower) components of the doublets now appear mixed with the charged ones (upper), the resulting $8 \times 8$ mass matrix having a total of four zero eigenvalues corresponding to the expected four Goldstone bosons arising from the full breaking of the gauge symmetry group.

The existence of diverse minima in the potential raises the possibility of tunneling between different vacua, and certainly the hypothetical existence of, for instance, a CB minimum deeper than a EW or CP one, could constitute a problem for the model. However, it has been shown that if a normal minimum exists, any $\mathrm{CP}$ or charge breaking solutions of the minimisation equations are necessarily saddle points which lie above the normal minimum [23-26]. In fact, it was possible to show that the value of the potential at normal vacua $\left(V_{N}\right), \mathrm{CP}$ stationary points $\left(V_{\mathrm{CP}}\right)$ or $\mathrm{CB}$ ones $\left(V_{\mathrm{CB}}\right)$ can be related to one another, for coexisting tree-level stationary points of these types. The following formulae have been established:

$$
\begin{aligned}
& V_{\mathrm{CB}}-V_{N}=\left(\frac{m_{H^{ \pm}}^{2}}{4 v^{2}}\right)_{N}\left[\left(v_{1} v_{2}^{\prime}-v_{2} v_{1}^{\prime}\right)^{2}+v_{1}^{2} v_{3}^{\prime 2}\right] \\
& V_{\mathrm{CP}}-V_{N}=\left(\frac{m_{A}^{2}}{4 v^{2}}\right)_{N}\left[\left(v_{1} \bar{v}_{2} \cos \theta-v_{2} \bar{v}_{1}\right)^{2}+v_{1}^{2} \bar{v}_{2}^{2} \sin ^{2} \theta\right],
\end{aligned}
$$

with the vevs for each possible stationary points defined in eqs. (2.5), (3.1) and (3.2), and the subscript " $N$ " refers that the masses $m_{H^{ \pm}}, m_{A}$ and the vev $v$ are computed at the normal stationary point. The terms within the square brackets are obviously positive thus, if $N$ is a minimum, its squared scalar masses will all be positive - and hence these expressions show that $V_{\mathrm{CB}}>V_{N}$ and $V_{\mathrm{CP}}>V_{N}$ when $N$ is a minimum. It is also easy to show that in that case both $C P$ and $C B$ stationary points would necessarily be saddle points. Analogously, if the potential is such that a $C P(C B)$ minimum occurs, any eventual normal or $C B(C P)$ stationary points would live above the minimum and be saddle points. Thus tunneling to deeper minima of a different nature is impossible in the 2HDM.

There is however another aspect of the $2 \mathrm{HDM}$ vacuum structure which sets it apart from the SM, to wit, in certain situations the minimization conditions allow for several non-equivalent normal stationary points [27]. Therefore, already at tree-level, there is the possibility of two (no more than two) normal minima coexisting in the potential, at different depths $[25,26]$. In other words, other than the normal vacuum with vevs given by eq. (2.5), for which one has $v_{1}^{2}+v_{2}^{2}=v^{2}=(246 \mathrm{GeV})^{2}$, there may exist a second normal vacuum $N^{\prime}$, with different vevs $\left\{v_{1}^{\prime}, v_{2}^{\prime}\right\}$. For this second minimum of the potential, the sum of the squared vevs takes a different value, smaller or larger than $(246 \mathrm{GeV})^{2}$. The two minima are not degenerate, in fact they verify $[28,29]$

$$
V_{N^{\prime}}-V_{N}=\frac{1}{4}\left[\left(\frac{m_{H^{ \pm}}^{2}}{v^{2}}\right)_{N}-\left(\frac{m_{H^{ \pm}}^{2}}{v^{2}}\right)_{N^{\prime}}\right]\left(v_{1} v_{2}^{\prime}-v_{2} v_{1}^{\prime}\right)^{2}
$$

where the quantity $\left(m_{H^{ \pm}}^{2} / v^{2}\right)$ is evaluated at each of the minima, $N$ and $N^{\prime}$. This raises the possibility that our vacuum, with $v=246 \mathrm{GeV}$, is not the deepest one - there is 
nothing, in eq. (3.5), which privileges the minimum $N$ over $N^{\prime}$, unlike what happened in eqs. (3.3) or (3.4). In fact, for certain regions of the $2 \mathrm{HDM}$ potential, $N^{\prime}$ may be found to be the global minimum of the model - a minimum where the exact same symmetries have been broken, but where all elementary particles have different masses. In that situation our universe could tunnel to this deeper minimum, with obvious catastrophic consequences.

The conditions under which this rather intriguing possibility arises were established in $[25,26,28,29]$. Defining the quantity

$$
k=\sqrt[4]{\frac{\lambda_{1}}{\lambda_{2}}}
$$

the necessary (but not sufficient) conditions for the softly broken $\mathbb{Z}_{2} 2 \mathrm{HDM}$ potential to have two minima are

$$
\begin{gathered}
m_{11}^{2}+k^{2} m_{22}^{2}<0 \\
\sqrt[3]{x^{2}}+\sqrt[3]{y^{2}} \leq 1
\end{gathered}
$$

where we have defined the variables $x$ and $y$ as

$$
\begin{aligned}
& x=\frac{4 k m_{12}^{2}}{m_{11}^{2}+k^{2} m_{22}^{2}} \frac{\sqrt{\lambda_{1} \lambda_{2}}}{\lambda_{345}-\sqrt{\lambda_{1} \lambda_{2}}}, \\
& y=\frac{m_{11}^{2}-k^{2} m_{22}^{2}}{m_{11}^{2}+k^{2} m_{22}^{2}} \frac{\sqrt{\lambda_{1} \lambda_{2}}+\lambda_{345}}{\sqrt{\lambda_{1} \lambda_{2}}-\lambda_{345}} .
\end{aligned}
$$

As shown in ref. [29], the EW vacuum " $N$ " ("our" minimum) is the global, true, minimum of the theory if and only if $D>0$, where the discriminant $D$ is a quantity given by

$$
D=m_{12}^{2}\left(m_{11}^{2}-k^{2} m_{22}^{2}\right)(\tan \beta-k) .
$$

Notice how, remarkably, the value of $D$ can, in principle, be obtained by experiments performed on "our" minimum, without any knowledge of the existence of $N^{\prime}$.

Let us again recall (see the discussion following eq. (2.9)) that if the minimisation conditions yield the solutions $N=\left\{v_{1}, v_{2}\right\}$ and $N^{\prime}=\left\{v_{1}^{\prime}, v_{2}^{\prime}\right\}$, they also include other "mirror" solutions, $\bar{N}=\left\{-v_{1},-v_{2}\right\}$ and $\bar{N}^{\prime}=\left\{-v_{1}^{\prime},-v_{2}^{\prime}\right\}$. This is a trivial consequence of the fact that the potential is invariant under a sign change of both doublets, $V\left(\Phi_{1}, \Phi_{2}\right)=V\left(-\Phi_{1},-\Phi_{2}\right)$, and apparently this has no physical consequences: the potential is degenerate at $N$ and $\bar{N}\left(N^{\prime}\right.$ and $\left.\bar{N}^{\prime}\right)$, and physics at these two minima is entirely identical. No physical differences whatsoever may arise from being at $N$ or $\bar{N}\left(N^{\prime}\right.$ or $\left.\bar{N}^{\prime}\right)$, because the only difference between both minima is the overall sign of both fields - no interference effects, for instance, will be sensitive to the sign change. The SM minimum of the Higgs potential, of course, is also degenerate with a continuum of other possible solutions - recall the shape of the tree-level SM Higgs potential, where infinitely many degenerate minima lie in a full circle. This is due to the fact that the SM minimum is determined by the equation $\langle|\Phi|\rangle=v / \sqrt{2}$, which yields a continuum of possible solutions, corresponding to different gauge choices for the Higgs doublet $\Phi$. However, for the $2 \mathrm{HDM}$ potential, each of the minima $N$ and $N^{\prime}$ is not degenerate with a continuum of other 
minima, but rather with another separate isolated minimum, $\bar{N}$ and $\bar{N}^{\prime}$ respectively. We emphasize these seemingly trivial aspects of the minimisation solutions because they may have dramatic consequences in the computation of tunneling rates, as will be discussed below in section 5 .

The mere existence of a deeper minimum is however no valid reason to exclude the values of the $2 \mathrm{HDM}$ scalar parameters which produce it. In fact, should the lifetime of the false vacuum be larger than the current age of the universe, such situation, however strange, would be phenomenologically acceptable. In [29] a quick estimate of the lifetime of $2 \mathrm{HDM}$ false vacua was undertaken, but even then several shortcomings of the calculation were pointed out: the fact that it was inspired by a single field tunneling computation, even though in the $2 \mathrm{HDM}$ the number of fields varying from minimum to minimum is larger; the use of a "thin-wall" approximation; the imposition of a bounce trajectory necessarily passing by an intermediate saddle point. A thorough study of the tunneling times between $\mathrm{EW}$ vacua is necessary to impose valid constraints on the $2 \mathrm{HDM}$ parameter space, and we will undertake it in the next sections.

\section{Tunneling and bounces}

In the present section we briefly review the theoretical background for the computation of the tunneling decay rate from a false vacuum to a true vacuum, starting from the one field case studied by Coleman and Callan [32,33], and extending then the result to the general $N$ field case.

Let us begin by considering the Euclidean action for a single component real scalar field $\phi$ :

$$
S[\phi]=\int d^{4} x\left[\frac{1}{2}\left(\partial_{\mu} \phi\right)^{2}+V(\phi)\right]
$$

where $V(\phi)$ is a potential with a local minimum (false vacuum) at $\phi=\phi_{\mathrm{fv}}$, and an absolute minimum (true vacuum) at $\phi=\phi_{\mathrm{tv}}$. In order to calculate the false vacuum lifetime we have to look for the so called bounce solution to the Euclidean Euler-Lagrange equation that have $O(4)$ symmetry and satisfy specific boundary conditions [32]. Denoting by $r$ the euclidean radial coordinate, $r=\sqrt{t_{E}^{2}+x^{2}}$, where $t_{E}=-i t$ is the imaginary time, the action (4.1) for $O(4)$ configurations takes the form

$$
S[\phi]=2 \pi^{2} \int_{0}^{\infty} d r r^{3}\left[\frac{1}{2}\left(\frac{d \phi}{d r}\right)^{2}+V(\phi)\right],
$$

while the equation of motion is

$$
\frac{d^{2} \phi}{d r^{2}}+\frac{3}{r} \frac{d \phi}{d r}=\frac{d V}{d \phi}
$$

and the above mentioned boundary conditions are:

$$
\phi(\infty)=\phi_{\mathrm{fv}} \quad \text { and }\left.\quad \frac{d \phi(r)}{d r}\right|_{r=0}=0 .
$$


As the bounce solution $\phi_{b}(r)$ in the $r \rightarrow \infty$ limit goes to $\phi_{\mathrm{fv}}$, the action (4.2) is infinite when calculated at $\phi(r)=\phi_{b}(r)$. However, for the calculation of the tunneling time we need to subtract to the bounce action the corresponding action calculated at the false vacuum $\phi_{\mathrm{fv}}: S\left[\phi_{b}\right]-S\left[\phi_{\mathrm{fv}}\right]$ (see below). Due to the asymptotic $(r \rightarrow \infty)$ behaviour of $\phi_{b}(r)[85,86]$, the subtracted action

$$
B \equiv S\left[\phi_{b}\right]-S\left[\phi_{\mathrm{fv}}\right]=2 \pi^{2} \int_{0}^{\infty} d r r^{3}\left[\frac{1}{2}\left(\frac{d \phi_{b}}{d r}\right)^{2}+V\left(\phi_{b}\right)-V\left(\phi_{\mathrm{fv}}\right)\right]
$$

is finite.

Denoting by $B_{K}$ and $B_{V}$ the kinetic and the potential terms in (4.5), it is easily shown that $B_{V}=-1 / 2 B_{K}$, so that $B=1 / 2 B_{K}=\frac{\pi^{2}}{2} \int_{0}^{\infty} d r r^{3}\left(\frac{d \phi_{b}}{d r}\right)^{2}$. Integrating now by parts and using the equation of motion (4.3), we get:

$$
B=-\frac{\pi^{2}}{2} \int_{0}^{\infty} d r r^{3} \frac{d V}{d \phi_{b}} \phi_{b}
$$

The general formula for the decay rate $\Gamma$ of the false vacuum is $[32,33]$ :

$$
\Gamma=D e^{-B}
$$

and $B$ is usually called the tunneling exponent. The exponential of $-B$ gives the "treelevel" contribution to the decay rate, while the prefactor $D$ contains the contributions from the quantum fluctuation determinant, including those coming from the zero modes.

Denoting by $T_{U}$ the age of the universe, and approximating the prefactor as $D \simeq$ $T_{U}^{3} \phi_{b}(0)^{4} \quad$ [87], the tunneling rate $\Gamma$ in (4.7) finally is:

$$
\Gamma=\left[T_{U}^{3} \phi_{b}(0)^{4}\right] e^{-B},
$$

where $\phi_{b}(0)$ is the value of the bounce at $r=0$, which is the center of the bounce. The tunneling time from the false to the true vacuum, i.e. the lifetime $\tau$ of the false vacuum, is then given by the inverse of $\Gamma: \tau=\Gamma^{-1}$. Naturally, if the false vacuum can decay towards more than one state, the tunneling rate $\Gamma$ is obtained by calculating the different rates $\Gamma_{i}$, so that: $\Gamma=\sum_{i} \Gamma_{i}$, and again $\tau=\Gamma^{-1}$.

The extension to the case with $N$ real fields $\phi_{i}, i=1, \ldots, N$ is straightforward. If we denote the fields with $\phi=\left(\phi_{1}, \phi_{2}, \ldots, \phi_{N}\right)$ and the potential as $V(\phi)$, the bounce configuration is a non-trivial solution of the coupled system of $N$ ordinary differential equation:

$$
\frac{d^{2} \phi_{i}}{d r^{2}}+\frac{3}{r} \frac{d \phi_{i}}{d r}=\frac{\partial V(\phi)}{\partial \phi_{i}}
$$

with boundary conditions

$$
\begin{aligned}
& \left.\frac{d \phi_{i}}{d r}\right|_{r=0}=0 \\
& \lim _{r \rightarrow \infty} \phi_{i}=\phi_{i}^{\mathrm{fv}},
\end{aligned}
$$


where $\phi_{i}=\phi_{i}^{\mathrm{fv}}$ are the values of the fields $\phi_{i}$ at the false vacuum. Following the same steps that lead to eq. (4.6), the action calculated at the bounce solution $\phi_{b}(r)=$ $\left(\phi_{1}(r), \ldots, \phi_{N}(r)\right)_{\text {bounce }}$ for the $N$ field case takes the form:

$$
B=-\frac{\pi^{2}}{2} \int_{0}^{\infty} d r r^{3}\left[\frac{d V(\phi)}{d \phi_{i}} \phi_{i}\right]_{\phi_{b}},
$$

where a sum over $i$ is implied.

Apart from very simple cases, the system (4.9) cannot be solved analytically and we have to rely on numerical methods to evaluate the bounce configurations. To this end, we used the public Wolfram Mathematica code developed in [42]. The latter solves the system (4.9) with the help of a multiple shooting method, exploiting the asymptotic behavior of the bounce solution for $r \rightarrow 0$ and $r \rightarrow \infty$ (that is known in both cases analytically). Finally, the tunneling rate is given by:

$$
\Gamma=T_{U}^{3}\left[\sum_{i} \phi_{i}^{2}(0)\right]^{2} e^{-B}
$$

As for the one field case, if the false vacuum can decay towards more than one state, $\Gamma$ is obtained by calculating the different rates $\Gamma_{i}: \Gamma=\sum_{i} \Gamma_{i}$ and $\tau=\Gamma^{-1}$.

For the $2 \mathrm{HDM}$ case, the two doublets have a total of eight real components, as seen in eq. (2.6). Therefore, in principle, the calculation of the bounce solution should involve all eight fields, which should contribute to the tunneling time shown in eq. (4.13). However, the gauge structure of the model allows a considerable simplification of this procedure. In fact, since the model has a $\mathrm{SU}(2) \times \mathrm{U}(1)$ gauge invariance, we are at liberty to choose a specific gauge, that allows us to remove several of the real components of the doublets. This is a well-known feature of the 2HDM (see, for instance, the demonstration of this possibility in section 5.8 of ref. [5]) which, in passing, is also the reason why the most generic vacua of the model can be cast into the form of eqs. (2.5), (3.1) and (3.2). In the end, we can choose to eliminate two of the upper components of the doublets (two charged fields) and one of the imaginary components of the lower part of the doublets, so that we are left with simplified doublets given by

$$
\Phi_{1}=\frac{1}{\sqrt{2}}\left(\begin{array}{c}
0 \\
\phi_{1}
\end{array}\right), \quad \Phi_{2}=\frac{1}{\sqrt{2}}\left(\begin{array}{c}
\phi_{4}+\mathrm{i} \phi_{5} \\
\phi_{2}+\mathrm{i} \phi_{3}
\end{array}\right),
$$

where for convenience we have renamed the real component fields.

For the CP-conserving potential of eq. (2.1) that we have been studying, the bounce equation (4.9) will allow a further simplification, involving only two of the above component fields, namely $\phi_{1}$ and $\phi_{2}$. In fact, let us consider the derivatives of the potential with respect to each of the $\phi_{i}$ that appear in the right-hand side of the bounce equation (4.9). These are given by

$$
\frac{\partial V}{\partial \phi_{1}}=\frac{1}{2}\left[2 m_{11}^{2}+\lambda_{1} \phi_{1}^{2}+\lambda_{3}\left(\phi_{2}^{2}+\phi_{3}^{2}+\phi_{4}^{2}+\phi_{5}^{2}\right)+\lambda_{4}\left(\phi_{2}^{2}+\phi_{3}^{2}\right)+\lambda_{5}\left(\phi_{2}^{2}-\phi_{3}^{2}\right)\right] \phi_{1}-m_{12}^{2} \phi_{2}
$$




$$
\begin{aligned}
\frac{\partial V}{\partial \phi_{2}} & =\frac{1}{2}\left[2 m_{22}^{2}+\lambda_{2}\left(\phi_{2}^{2}+\phi_{3}^{2}+\phi_{4}^{2}+\phi_{5}^{2}\right)+\left(\lambda_{3}+\lambda_{4}+\lambda_{5}\right) \phi_{1}^{2}\right] \phi_{2}-m_{12}^{2} \phi_{1} \\
\frac{\partial V}{\partial \phi_{3}} & =\frac{1}{2}\left[2 m_{22}^{2}+\lambda_{2}\left(\phi_{2}^{2}+\phi_{3}^{2}+\phi_{4}^{2}+\phi_{5}^{2}\right)+\left(\lambda_{3}+\lambda_{4}-\lambda_{5}\right) \phi_{1}^{2}\right] \phi_{3} \\
\frac{\partial V}{\partial \phi_{4}} & =\frac{1}{2}\left[2 m_{22}^{2}+\lambda_{2}\left(\phi_{2}^{2}+\phi_{3}^{2}+\phi_{4}^{2}+\phi_{5}^{2}\right)+\lambda_{3} \phi_{1}^{2}\right] \phi_{4} \\
\frac{\partial V}{\partial \phi_{5}} & =\frac{1}{2}\left[2 m_{22}^{2}+\lambda_{2}\left(\phi_{2}^{2}+\phi_{3}^{2}+\phi_{4}^{2}+\phi_{5}^{2}\right)+\lambda_{3} \phi_{1}^{2}\right] \phi_{5} .
\end{aligned}
$$

Notice how in the three last equations the fields $\phi_{3}, \phi_{4}$ and $\phi_{5}$ factorize, and how that does not occur for the derivatives of the potential with respect to $\phi_{1}$ and $\phi_{2}$. This leads to bounce equations for each of the $\phi_{i}$ of the following form:

$$
\begin{aligned}
& \frac{d^{2} \phi_{1}}{d r^{2}}+\frac{3}{r} \frac{d \phi_{1}}{d r}=f_{1}\left(\phi_{1}, \ldots \phi_{5}\right) \phi_{1}-m_{12}^{2} \phi_{2} \\
& \frac{d^{2} \phi_{2}}{d r^{2}}+\frac{3}{r} \frac{d \phi_{2}}{d r}=f_{2}\left(\phi_{1}, \ldots \phi_{5}\right) \phi_{2}-m_{12}^{2} \phi_{1} \\
& \frac{d^{2} \phi_{3}}{d r^{2}}+\frac{3}{r} \frac{d \phi_{3}}{d r}=f_{3}\left(\phi_{1}, \ldots \phi_{5}\right) \phi_{3} \\
& \frac{d^{2} \phi_{4}}{d r^{2}}+\frac{3}{r} \frac{d \phi_{4}}{d r}=f_{4}\left(\phi_{1}, \ldots \phi_{5}\right) \phi_{4} \\
& \frac{d^{2} \phi_{5}}{d r^{2}}+\frac{3}{r} \frac{d \phi_{5}}{d r}=f_{5}\left(\phi_{1}, \ldots \phi_{5}\right) \phi_{5},
\end{aligned}
$$

where the functions $f_{i}$ can be read from eqs. (4.15)-(4.19). These equations must be solved with the boundary conditions (4.10) and (4.11). In our case, for which both the true and false vacua of the $\mathrm{CP}$ conserving potential are themselves $\mathrm{CP}$ and charge conserving, the boundary condition (4.11) always implies $\phi_{3}(\infty)=\phi_{4}(\infty)=\phi_{5}(\infty)=0$ at any vacua.

We observe that there is a fundamental difference between the bounce equations for $\left\{\phi_{1}, \phi_{2}\right\}$ and those for $\left\{\phi_{3}, \phi_{4}, \phi_{5}\right\}$ - namely, in the right-hand side of the latter equations the factorization of the fields $\phi_{3}, \phi_{4}$ and $\phi_{5}$ implies that the trivial solutions $\phi_{3}(r)=0$, $\phi_{4}(r)=0$ and $\phi_{5}(r)=0$ exist. Moreover, they respect the above-mentioned boundary conditions, and thus are acceptable bounce solutions. On the contrary, in the right-hand side of the first two equations there is an extra term linear in the fields $\phi_{1}$ and $\phi_{2}$. And, though the trivial solutions $\phi_{1}(r)=0$ and $\phi_{2}(r)=0$ also satisfy eqs. (4.20) and (4.21), they do not comply with the boundary conditions at infinity for these two fields, which are of the form $\phi_{1}(\infty)=v_{1}$ and $\phi_{2}(\infty)=v_{2}$ with non-zero values for the false vacua vevs $v_{1}$ and $v_{2}:{ }^{4}$ thus they are not bounce solutions.

This strongly suggests that the bounce solutions connecting the true and false vacua have the profiles $\phi_{3}(r), \phi_{4}(r)$ and $\phi_{5}(r)$ identically vanishing in the whole range for $r$, from 0 to $\infty$. This would imply that the original 2 HDM 8 -field bounce calculation reduces to a 2-field problem. In fact, in all the hundreds of thousands of cases that we have studied

\footnotetext{
${ }^{4}$ Notice how the soft breaking term $m_{12}^{2}$ in the potential prevents solutions of the minimisation conditions of eq. (2.9) with any of the vevs equal to zero.
} 
numerically (see section 6), we have always verified that only $\phi_{1}(r)$ and $\phi_{2}(r)$ have nontrivial profiles, while $\phi_{3}(r), \phi_{4}(r)$ and $\phi_{5}(r)$ always vanish. ${ }^{5}$

This is not merely a mathematical property of the bounce equations (4.9), but rather it is dictated by the physics of the model. To illustrate this point, let us consider for the moment the Complex 2HDM (C2HDM) [88-97], where no CP symmetry is imposed on the potential of eq. (2.2). In this generalisation, both parameters $m_{12}^{2}$ and $\lambda_{5}$ can be complex although one of these phases can always be absorbed into one of the fields. We are then left with a single complex parameter in the potential, which we choose as the soft breaking term. Let us then write $m_{12}^{2}=\left|m_{12}^{2}\right| \exp ^{\theta_{12}}$. It is well known [27] that this potential may have coexisting minima as well - now, however, there is the possibility that in one of these minima the vevs of the doublets are real (as in eq. (2.5)) and in the other the vevs have a relative complex phase (as in eq. (3.1)). But since the potential explicitly breaks the CP symmetry due to the presence of the phase $\theta_{12}$, both of these vacua are CP breaking, even if the vevs are real. For the C2HDM potential with complex $m_{12}^{2}$, then, the derivatives of the potential with respect to $\phi_{i}$ are slightly modified, and the bounce equations (4.20)-(4.24) become

$$
\begin{aligned}
\frac{d^{2} \phi_{1}}{d r^{2}}+\frac{3}{r} \frac{d \phi_{1}}{d r} & =f_{1}\left(\phi_{1}, \ldots \phi_{5}\right) \phi_{1}-\left|m_{12}^{2}\right|\left(\phi_{2} \cos \theta_{12}-\phi_{3} \sin \theta_{12}\right) \\
\frac{d^{2} \phi_{2}}{d r^{2}}+\frac{3}{r} \frac{d \phi_{2}}{d r} & =f_{2}\left(\phi_{1}, \ldots \phi_{5}\right) \phi_{2}-\phi_{1}\left|m_{12}^{2}\right| \cos \theta_{12} \\
\frac{d^{2} \phi_{3}}{d r^{2}}+\frac{3}{r} \frac{d \phi_{3}}{d r} & =f_{3}\left(\phi_{1}, \ldots \phi_{5}\right) \phi_{3}+\phi_{1}\left|m_{12}^{2}\right| \sin \theta_{12} \\
\frac{d^{2} \phi_{4}}{d r^{2}}+\frac{3}{r} \frac{d \phi_{4}}{d r} & =f_{4}\left(\phi_{1}, \ldots \phi_{5}\right) \phi_{4} \\
\frac{d^{2} \phi_{5}}{d r^{2}}+\frac{3}{r} \frac{d \phi_{5}}{d r} & =f_{5}\left(\phi_{1}, \ldots \phi_{5}\right) \phi_{5} .
\end{aligned}
$$

Comparing the system of equations (4.20)-(4.24) with the corresponding system (4.25)(4.29) we observe that, while the two last equations remain unchanged, the right hand side of the third equation contains an additional term that does not factorize $\phi_{3}$ (further, the non-factorized terms in eqs. (4.25)-(4.26) have also changed). Thus, we no longer expect a trivial profile for the bounce solution $\phi_{3}(r)$. Clearly, the appearance of the additional term in the bounce equation for $\phi_{3}$, which we recall is the complex neutral component of the second doublet, depends on the presence of the explicitly $\mathrm{CP}$ breaking phase $\theta_{12}$ : the different physics described by the $\mathrm{C} 2 \mathrm{HDM}$ induces a different structure in the bounce equations. At this point, we consider many different choices for the parameters of the C2HDM potential in which coexisting minima occur. These points are chosen such that the false vacuum has real vevs, while the vevs of the true minimum have a relative complex phase. Computing the bounce solution for this parameter space, our expectation for the bounce profiles is fully confirmed: for this new model, $\phi_{1}, \phi_{2}$ and $\phi_{3}$ are non trivial profiles, while $\phi_{4}$ and $\phi_{5}$ vanish as before. We see a particular example of this behaviour in figure 1 , where we plot the different fields of the bounce solution $\phi_{i}$ as a function of $r$. We remind

\footnotetext{
${ }^{5}$ Notice however that we do not possess a full analytical demonstration of this property.
} 


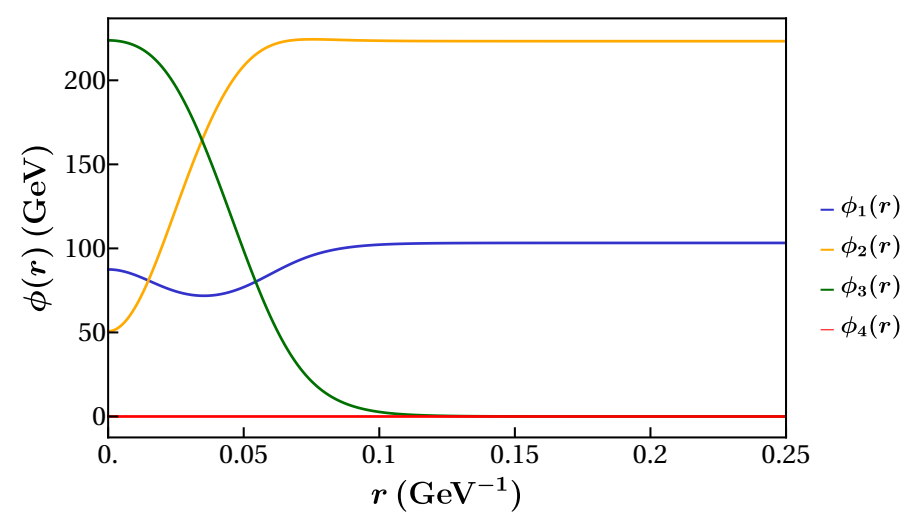

Figure 1. Bounce solution $\left(\phi_{1}, \phi_{2}, \phi_{3}, \phi_{4}\right)$ for the C2HDM. The fifth bounce for $\phi_{5}$ is identical to that for $\phi_{4}$.

the reader that in this plot the fields tend at $r \rightarrow \infty$ to the false vacua vevs - and thus $\phi_{3}$ in that limit vanishes, as expected. As opposed to what happened in the $\mathrm{CP}$ conserving potential, however, $\phi_{3}(r)$ is no longer vanishing everywhere. In particular, we observe that at $r=0$ it is taking a non-zero value, thus contributing (as well as the other non-zero components of the bounce) to the evaluation of the tunneling time in eq. (4.13).

It is worth stressing at this stage that this change in the behaviour of $\phi_{3}(r)$ is due to the different physics described by the two potentials. Only due to the explicit $\mathrm{CP}$ violation of the $\mathrm{C} 2 \mathrm{HDM}$ can $\phi_{3}$ have a non-trivial profile, while explicit $\mathrm{CP}$ conservation forces this component of the bounce to vanish for all values of $r$. Further, notice that for both potentials $\phi_{4}$ and $\phi_{5}$ are always vanishing - which is to be expected on physical grounds, since no charge breaking can occur in either of the models when a normal minimum exists.

\section{$5 \quad$ Tunneling to degenerate vacua}

At this stage, and before we embark on scans of the 2HDM parameter space, let us discuss a novel aspect of the tunneling calculations which arise in this model. As we have emphasised previously, if the minimisations conditions (2.9) have a solution of the form $\left\{v_{1}, v_{2}\right\}$, they also include the solutions $\left\{-v_{1},-v_{2}\right\}$. The same happens for the second, non degenerate minimum $N^{\prime}$, which corresponds to vevs of the form $\left\{v_{1}^{\prime}, v_{2}^{\prime}\right\}$.

Let now $N \equiv\left\{v_{1}, v_{2}\right\}$ and $\bar{N} \equiv\left\{-v_{1},-v_{2}\right\}$ be the false vacua of the model, and assume that "our" vacuum corresponds to $N$. The universe may now tunnel to two degenerate true vacua, $N^{\prime}=\left\{v_{1}^{\prime}, v_{2}^{\prime}\right\}$ and $\bar{N}^{\prime}=\left\{-v_{1}^{\prime},-v_{2}^{\prime}\right\}$. Since $N^{\prime}$ and $\bar{N}^{\prime}$ describe exactly the same physics, one could expect that there would be absolutely no difference between the tunneling rates from $N$ to either $N^{\prime}$ or $\bar{N}^{\prime}$. This, remarkably, is not the case.

In order to understand this critical point, let us consider a specific example, for which the parameters of the $2 \mathrm{HDM}$ potential (2.2) are chosen to be

$$
\begin{aligned}
& m_{11}^{2}=-23519.8, \quad m_{22}^{2}=-10249.6, \quad m_{12}^{2}=-6145.98 \quad\left(\mathrm{GeV}^{2}\right) \\
& \lambda_{1}=4.59143, \quad \lambda_{2}=0.388928, \quad \lambda_{3}=1.79703, \\
& \lambda_{4}=-1.80544, \quad \lambda_{5}=-0.481738 \text {. }
\end{aligned}
$$


This choice of parameters yields a maximum $M$ at field values $M \equiv\left\{\phi_{1}, \phi_{2}\right\}=\{0,0\}$ and the following minima (all vevs in $\mathrm{GeV}$ ),

$$
\begin{aligned}
N & \equiv\{97.3767,225.907\}, & \bar{N} & \equiv\{-97.3767,-225.907\} \\
N^{\prime} & \equiv\{162.491,-319.463\}, & \bar{N}^{\prime} & \equiv\{-162.491,319.463\} .
\end{aligned}
$$

We also have two couples of saddle points,

$$
\begin{array}{ll}
S_{1} \equiv\{43.6574,221.06\}, & \bar{S}_{1} \equiv\{-43.6574,-221.06\} \\
S_{2} \equiv\{95.5578,48.8458\}, & \bar{S}_{2} \equiv\{-95.5578,-48.8458\} .
\end{array}
$$

If we now calculate the bounce solutions for the transitions from $N$ to $N^{\prime}$ and from $N$ to $\bar{N}^{\prime}$, and assuming for the sake of argument that only one of these transitions was possible, we would obtain the following tunneling times (see eq. (4.13)),

$$
\begin{aligned}
& \tau\left(N \rightarrow N^{\prime}\right) \simeq 8 \times 10^{2131} T_{U}, \\
& \tau\left(N \rightarrow \bar{N}^{\prime}\right) \simeq 2 \times 10^{-113} T_{U},
\end{aligned}
$$

where $T_{U}$ is the current age of the universe. ${ }^{6}$ If one were to only consider the transition $N \rightarrow N^{\prime}$ one would conclude that the false vacuum $N$ was absolutely stable - whereas the second transition, $N \rightarrow \bar{N}^{\prime}$, instead shows $N$ to be incredibly unstable, having decayed to $\bar{N}^{\prime}$ almost immediately after the Big Bang. The discrepancy between the tunneling times for both transitions is astonishing, all the more so because the lower minima $N^{\prime}$ and $\bar{N}^{\prime}$ are degenerate and describe exactly the same physics! Thus one might naïvely expect that there should be no difference in the tunneling rate from $N$ to either of them - after all the difference in the value of the potential between $N$ and $N^{\prime}$ or between $N$ and $\bar{N}^{\prime}$ is exactly the same, and given by eq. (3.5). How can such a difference in behaviour be explained? The fundamental reason is extremely simple to understand, and lies in the landscape of minima and saddle points yielding very different possible paths for tunneling between $N$ and $N^{\prime}$ or $\bar{N}^{\prime}$. This may be seen in figure 2 , where we illustrate, in the $\left\{\phi_{1}, \phi_{2}\right\}$ plane, the locations of all extrema of the potential listed above. Notice how $N$ is not equally distant from $N^{\prime}$ and $\bar{N}^{\prime}$; notice also, and perhaps even more importantly, that the path from $N$ to both of the lower minima passes close to a different landscape of saddle points - whereas from $N$ to $\bar{N}^{\prime}$ there is a saddle point almost at the beginning, to $N^{\prime}$ the first saddle point is further away. Also, the steepest descent from $N$ to $N^{\prime}$ is possibly "deviated" by the several remaining saddle points and the maximum along the way, which would explain the much larger tunneling time found, whereas the path to $\bar{N}^{\prime}$ seems much more "direct". To further drive in this point, consider figure 3, where we show 3D plots illustrating the shape of the potential along the (seemingly) shortest path from $N$ to both $N^{\prime}$ and $\bar{N}^{\prime}$ — these

\footnotetext{
${ }^{6}$ The factors multiplying $T_{U}$ in eq. (5.4) are either ridiculously low or ridiculously large. Recall, however, that they stem from the exponential of the action (4.6), which is quartic in the fields. Since in the bounce solution the fields acquire values of the order of the hundreds of $\mathrm{GeV}$, the action will be either positive or negative, but always include terms of the order of $10^{8} \mathrm{GeV}^{4}$, which leads to such stunning numbers as those in eq. (5.4).
} 


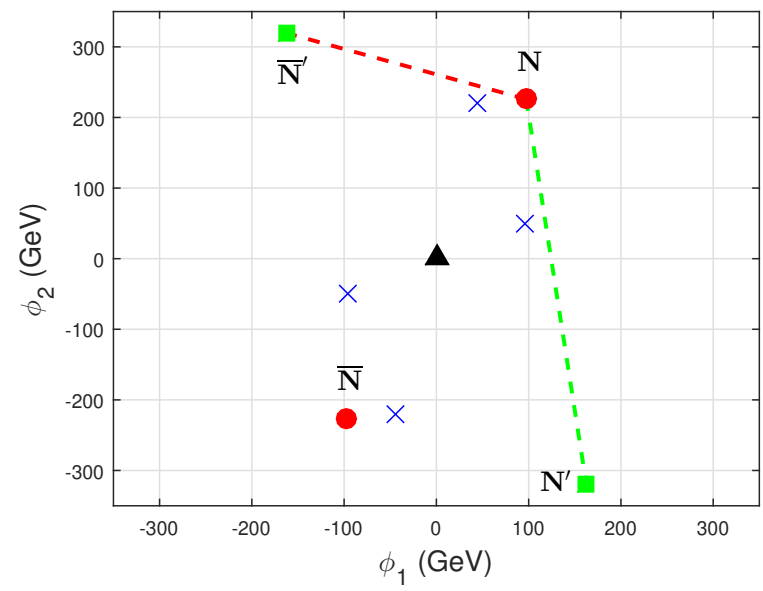

Figure 2. Location of all extrema of the $2 \mathrm{HDM}$ potential for the choice of parameters in (5.1). Saddle points are marked with " $\times$ ", the maximum of the potential, at $(0,0)$, with a black triangle. The false minima are marked with red circles, the true ones with green squares. The lines connecting $N$ to $N^{\prime}$ and $\bar{N}^{\prime}$ illustrate how different the paths between these minima may be.
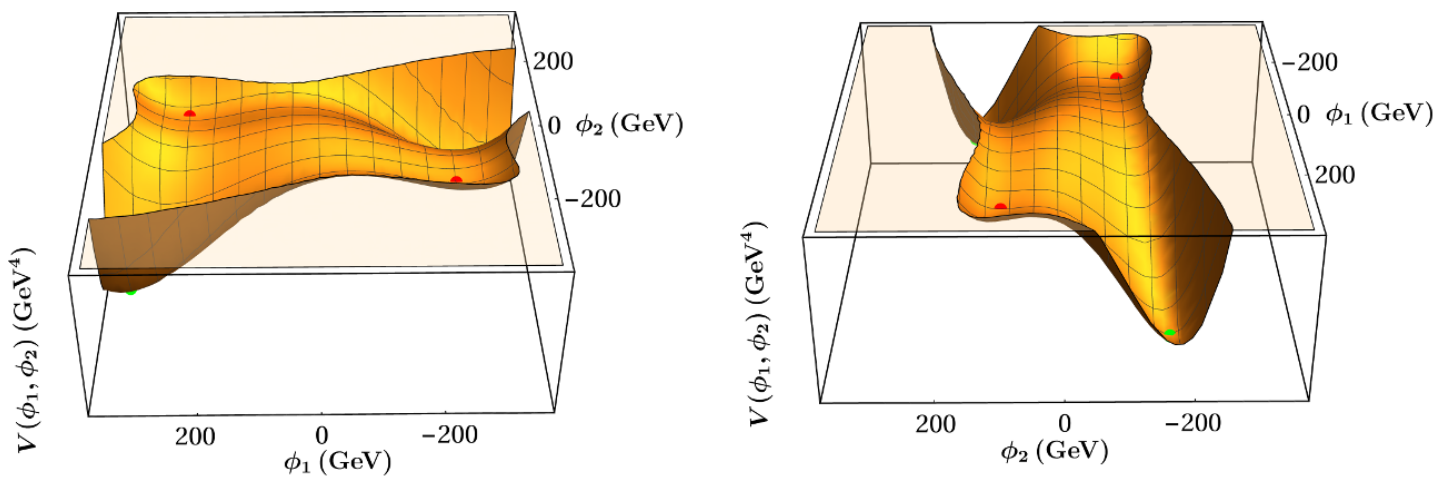

Figure 3. Left panel: plot of the potential $V\left(\phi_{1}, \phi_{2}\right)$ of eq. (2.2) for the parameters given of eq. (5.1). Right panel: the same potential rotated anticlockwise by 90 degrees. The left panel better shows the decay path from $N$ to $N^{\prime}$; the right panel from $N$ to $\bar{N}^{\prime}$. The path connecting $N$ while $N^{\prime}$ is longer than the path connecting $N$ with $\bar{N}^{\prime}$. False minima marked in red, true ones in green.

images show that, even though the difference in depth of the potential is exactly the same between $N$ and $N^{\prime}$ or between $N$ and $\bar{N}^{\prime}$, it is quite clearly easier for the latter transition to occur than the former. And in fact the bounce solutions obtained in the transition from $N$ to $N^{\prime}$ (which we now call " $B 1$ ") and from $N$ to $\bar{N}^{\prime}$, (" $B 2$ ") are quite different, as can be appreciated in figure 4 . In this plot we present the evolution with the radial coordinate $r$ (introduced in section 4) of the two bounce profiles for the fields $\phi_{1}$ and $\phi_{2}$ found for the specific example we have been considering. Notice how the solutions, $B 1$ and $B 2$, converge for large values of $r$ to the same values - which are the values of the vevs at the false vacuum $N$, as was to be expected. However, the values of the fields $\phi_{i}$ at $r=0$ diverge significantly, assuming even opposite signs. Recall that at $r=0$ the bounce solution is 


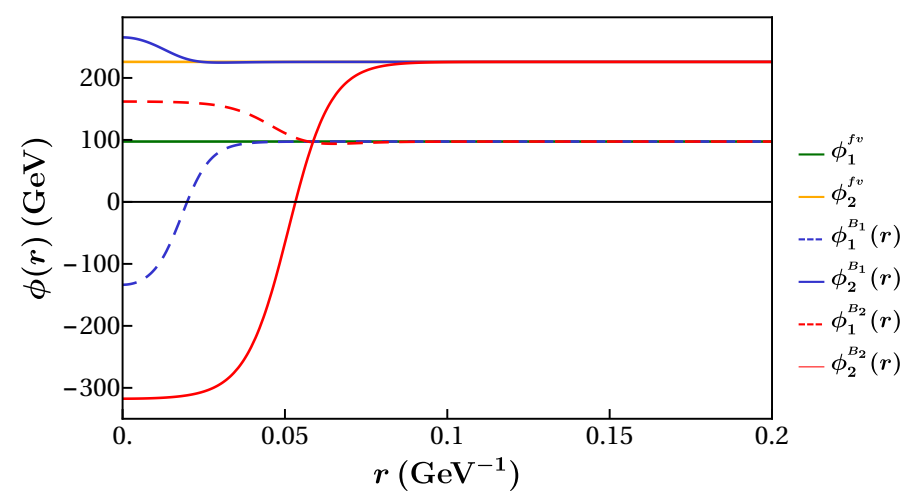

Figure 4. Bounce solutions for fields $\phi_{1}$ and $\phi_{2}$ for the transitions from $N$ to $N^{\prime}(B 1)$ and from $N$ to $\bar{N}^{\prime}(B 2)$. In both cases the fields $\phi_{i}$ tend to the same values at $r \rightarrow \infty$, i.e. the values of the fields at the false vacuum $N, \phi_{1}^{\mathrm{fv}}$ e $\phi_{2}^{\mathrm{fv}}$. But at $r=0$ the fields assume different values, close to the vevs at each of the degenerate true vacua.

found for values of the fields "close" to the true vacuum of the theory. Hence we find that, for the bounce $B 1, \phi_{1}$ assumes a large negative value, $\simeq-130 \mathrm{GeV}$ and $\phi_{2}$ a large positive one, $\simeq 260 \mathrm{GeV}$ - notice how these values for the bounce are close to the vevs of the true vacuum $\bar{N}^{\prime}(\sim-162, \sim 320 \mathrm{GeV})$. Likewise, the values found for the bounce solution $B 2$ are close to the vevs found for the other lower vacuum, $N^{\prime}$. Thus, despite the fact that both $N^{\prime}$ and $\bar{N}^{\prime}$ are degenerate and at the same relative depth to $N$, the bounce solutions for the two possible transitions are very different, and in fact lead to very different values for the bounce action $S\left[\phi_{b}\right]$ from eq. (4.6) - and hence to the two extremely different lifetime values found.

If the potential has, from $N$, two possible "decay channels", then its decay rate, $\Gamma$, will be given by

$$
\Gamma=\Gamma\left(N \rightarrow N^{\prime}\right)+\Gamma\left(N \rightarrow \bar{N}^{\prime}\right)=\frac{1}{\tau\left(N \rightarrow N^{\prime}\right)}+\frac{1}{\tau\left(N \rightarrow \bar{N}^{\prime}\right)}
$$

with the "partial" tunneling times from eq. (5.4). Thus, the lifetime $\tau$ of the false vacuum $N$ will obviously be

$$
\tau=\frac{1}{\Gamma}=\left(\frac{1}{\tau\left(N \rightarrow N^{\prime}\right)}+\frac{1}{\tau\left(N \rightarrow \bar{N}^{\prime}\right)}\right)^{-1} \simeq 2 \times 10^{-113} T_{U}
$$

where in analogy with nuclear decays, when one of the decay channels is much faster than the other, it dominates over the total lifetime. The conclusion to draw from this particular example is simple: both degenerate lower vacua must be considered for the calculation of the tunneling time, and the stability of the false vacuum may depend crucially on which of the true vacua it is decaying into. We have verified that differences in tunneling times to true degenerate vacua can be as extreme as those presented in eq. (5.4) for many choices of parameters in the potential, though not always. For many other regions of parameter space, though the two possible decay rates may differ, they do not affect qualitatively the 
overall stability of the false vacuum. Meaning, in many cases, if the tunneling time to one of the lower vacua is, say, much larger (smaller) than $T_{U}$, the other tunneling time, while possibly very different, will also be much larger (smaller) than $T_{U}$. Nonetheless, as we will shortly see, for certain regions the computation of $\tau$ taking into account the existence of both possible true vacua increased the number of dangerous false vacua by as much as $50 \%$.

\section{$6 \quad 2 \mathrm{HDM}$ numerical scans}

The physics arguments of section 4 show that the tunneling rate calculation can be reduced, for the CP conserving potential of eq. (2.2), to a two-field problem. Nonetheless we performed extensive numerical checks, comparing eight-field calculations with two-field ones, and no differences were ever found. Also, in section 5 we have shown the importance of computing the tunneling rates to both degenerate true vacua. Armed with these two important theoretical insights, we can proceed to an extensive scan of the $2 \mathrm{HDM}$ parameter space. Our goal is to ascertain how much of that parameter space should be avoided due to tunneling times shorter than the age of the universe.

We have chosen to work in models type I and II (for the remaining types of Yukawa interactions the conclusions reached would certainly be very similar). All parameter scans presented in this section are such that:

- They include at least one (CP conserving) minimum with $v=246 \mathrm{GeV}$ and $m_{h}=$ $125 \mathrm{GeV}$.

- All theoretical and experimental results mentioned in section 2.3 are satisfied. In particular, we demanded that all $\mu_{X}$ ratios (defined in eq. (2.12)) be within $30 \%$ of their expected SM value of 1 .

- $1 \leq \tan \beta \leq 30$ and $-\pi / 2 \leq \alpha \leq \pi / 2$.

- The mass of the heavier CP-even scalar $H$ is chosen in the interval between 130 and $700 \mathrm{GeV}$. The mass of the pseudoscalar $A$ is chosen between 100 and $700 \mathrm{GeV}$. For the charged mass, its lower bound is $100 \mathrm{GeV}$ for model type I and $580 \mathrm{GeV}$ for model type II (the difference due to flavour physics constraints described in section 2.3). The upper bound for the charged mass is again $700 \mathrm{GeV}$.

- The soft breaking parameter $m_{12}^{2}$ is taken with both signs, and magnitude below roughly $(500 \mathrm{GeV})^{2}$.

These parameter scans are not meant to be exhaustive - we merely wish to sample representative regions of the $2 \mathrm{HDM}$ parameter space to illustrate the possible impact that tunneling times to deeper vacua lower than the age of the universe may have. We now consider different scenarios. 

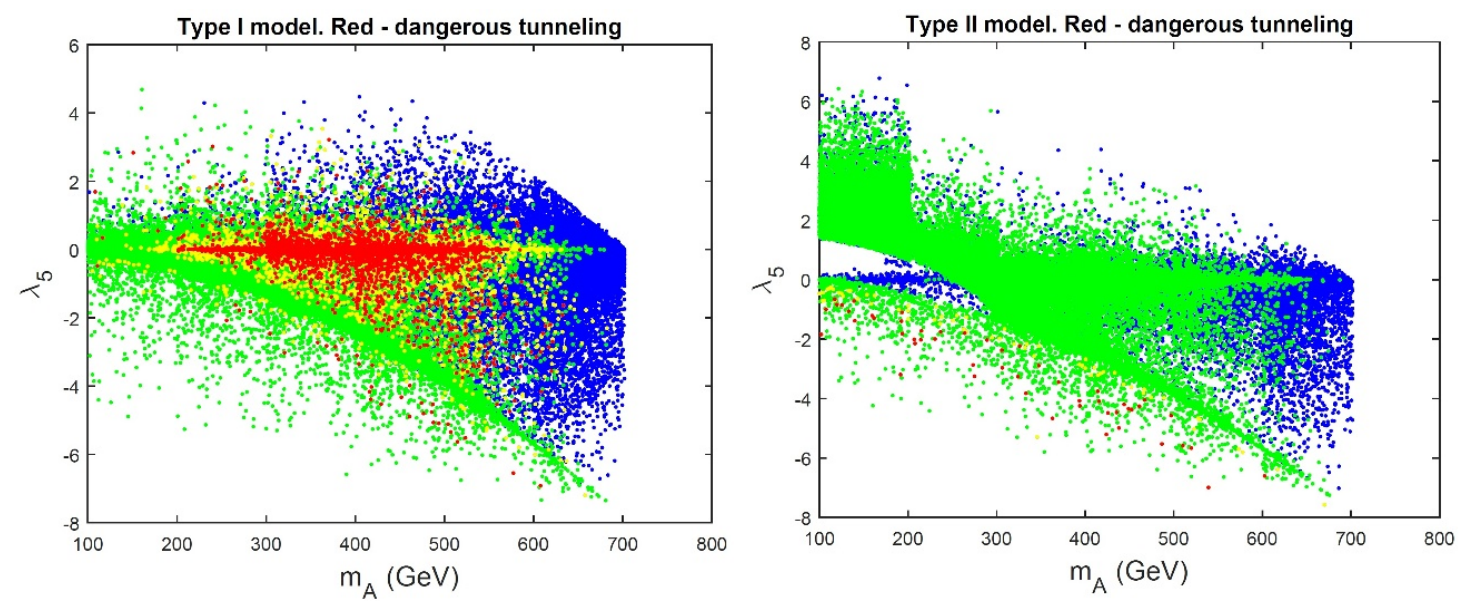

Figure 5. Scatter plot of $\lambda_{5}$ vs. $m_{A}$ for a general scan on the parameter space of a type I (left) and type II (right) 2HDM. In blue, all points generated which conform to theoretical and experimental constraints; in green, the subset of those for which two normal vacua are possible; in yellow, the subset of those for which $D<0$ and thus the EW vacuum may be the false one; and in red, those points for which the tunneling time to the true vacuum is smaller than the age of the universe.

\subsection{General scans for type I and II models}

To illustrate the possible relevance of false vacua exclusion (due to low tunneling times) in general scans of parameter space, we generated large datasets (over 100000 different combinations of parameters) for models type I and II.

In figure 5 we show the result of our analysis, by plotting the values of $\lambda_{5} v s$ the pseudoscalar mass $m_{A}$. The colour code in these plots is such that:

- In blue we present all points generated which satisfy the theoretical and experimental constraints explained above. Notice that other colours are superimposed on top of the blue points. Or, in other words, the green, yellow, red points are a subset of the blue ones.

- The green points correspond to the subset of the blue ones for which the two CPconserving minima conditions of eqs. (3.7) and (3.8) are satisfied. Recall that those conditions are necessary ones, but not sufficient, and therefore not all green points will truly correspond to the existence of two minima - in fact, that happens typically for only half of these points. Notice the disproportion in size of the green region compared to the blue one - dual minima in the $2 \mathrm{HDM}$ potential are, in general, a rare occurrence.

- In yellow we show the subset of the green points for which the discriminant $D$ from eq. (3.10) is negative - that is, the points for which, if two minima exist, "our" electroweak vacuum with $v=246 \mathrm{GeV}$ will not be the deeper one. ${ }^{7}$

\footnotetext{
${ }^{7}$ See also [98].
} 
- Finally in red, the subset of the yellow points for which: (a) two minima exist, (b) "our" electroweak vacuum is not the global minimum and (c) the tunneling time from "our" vacuum to the deeper true vacuum is less than the age of the universe.

The visible blue points in these figures are clearly safe combinations of parameters, for which the EW vacuum is not only safe but also unique. Several comments are in order to better interpret these plots. First, please take into account the fact that these plots are dense in each of the colours - in other words, in the middle of the green, or yellow, or red points there are blue ones. Thus the red regions are not wholly excluded - though dangerous tunneling times seem to be found for specific areas in the $m_{A}-\lambda_{5}$ plane, those areas will in general also include perfectly acceptable blue (green, yellow) points for which there might not even be two minima. Second, there is no obvious pattern to the green, yellow or red regions - the existence of two minima, and dangerous tunneling times for the acceptable EW vacuum, depends on non-trivial relations between the potential's parameters, which are difficult to visualize in this 2-dimensional slice of what is in truth an 8-dimensional parameter space. Third, in general it seems easier to find two minima (and dangerous short-lived vacua) in model type I than in II. This is a consequence of the hard bound on the charged Higgs mass in model type II which arises from $b \rightarrow s \gamma$ constraints - this bound tends to privilege higher, positive, values of $m_{12}^{2}$, for which the discriminant $D$ is usually found to be positive (and thus "our" EW vacuum is the global minimum of the model).

To illustrate the frequency with which dangerous vacua are found in this blind scan, consider the results shown above for type I: the total number of generated (blue) points conforming to all theory and experimental constraints was above 120000; of these, roughly 21500 (green) points were found which might have two minima (satisfying eqs. (3.7) and (3.8)) - in fact, of those, two minima were found only for over 11000 points. The (yellow) points with $D<0$, with possible local EW vacua with $v=246 \mathrm{GeV}$, totalled almost 9500, and out of these over 4200 were found for which the tunneling time to the global $(v \neq 246 \mathrm{GeV})$ minimum is inferior to $T_{U}$. Thus the percentage of points of the initial parameter space excluded on tunneling time arguments is about 3.5\%. For model type II, a similar accounting yields a percentage of excluded points of roughly $0.2 \%$.

The distribution of dangerous (red) points in figure 5 is clearly not homogenous, and the percentages of excluded points found in the previous paragraph are therefore not to be interpreted as, for instance, $3.5 \%$ of type I parameter space being ruled out on low tunneling times grounds. In fact, while certain regions of $2 \mathrm{HDM}$ parameter space are completely safe (the blue points visible in figure 5, for instance), others may yield a far larger percentage of dangerous minima than the numbers quoted above. To illustrate this let us now consider a few benchmark scenarios.

\subsection{First benchmark scenarios: safe regions}

As discussed above, the distribution of parameter space points for which dangerous shortlived false vacua occur in the $2 \mathrm{HDM}$ is not uniform. The regions of parameter space which conform to equations (3.7), (3.8) or have the discriminant (3.10) negative are usually not easily visualized in 2-dimensional slices, and as explained in section 5, the tunneling time 
to lower vacua may depend crucially on the landscape of saddle points in field space and this will also depend in a non-trivial manner on the numerical values of the couplings, affecting the number of possible solutions of the minimisation conditions of eq. (2.9). In the present subsection, we will give two examples of parameter choices for which, due to different reasons, the EW vacuum is perfectly safe. In all cases to follow we study model type I, and fix six of the parameters of the model, allowing two others to vary in such a manner as to comply with theory and experimental constraints. Since we wish to have a physically interesting EW vacuum, we chose to specify the values of (in principle) observable $2 \mathrm{HDM}$ parameters, rather than the couplings of the potential in eq. (2.2). To that end, we of course chose the value of $v=246 \mathrm{GeV}$ and $m_{h}=125 \mathrm{GeV}$ for the EW vacuum, and then proceed to select, for each benchmark scenario, the masses $m_{H}$ and $m_{H^{ \pm}}$, the value of $\tan \beta$ and of $\sin (\beta-\alpha)$ - thus, indirectly, the value of $\alpha$. We chose $\sin (\beta-\alpha)$ because this quantity is already quite constrained to be close to unity by LHC data.

The $2 \mathrm{HDM}$ parameter scan we undertake therefore considers these six parameters fixed and then proceeds to choosing random values for two others, which we chose to be $\lambda_{5}$ and $m_{A}{ }^{8}$ Each selection of parameters is then verified for theory and experimental constraints, and if all are obeyed a satisfactory EW vacuum is found. A posteriori the existence of a second minimum is checked, and if that second minimum is the global one, the tunneling time to the true vacuum is computed.

Decoupling scenario. As a first example, we have chosen $m_{H}=600 \mathrm{GeV}, m_{H^{ \pm}}=$ $700 \mathrm{GeV}, \tan \beta=1$ and $\sin (\beta-\alpha)=0.99$. Though $\lambda_{5}$ was allowed to vary between -10 and 10 , only values in the window between $\sim-6.3$ and $\sim-2.3$ were found after all constraints applied. ${ }^{9}$ Likewise, the pseudoscalar mass is found to be constrained between roughly 620 and $705 \mathrm{GeV}$. It is well known that the electroweak precision constraints (namely the bounds on the Peskin-Takeushi parameters $S, T$ and $U$ ) force the extra scalar masses to be close in the high mass range, so these results are not surprising.

The high values for the extra scalar masses coupled with the fact that $\sin (\beta-\alpha)$ is extremely close to $1,{ }^{10}$ means we are well within a decoupling regime for the model. And of course, one of the possible explanations for the current LHC results is the decoupling of all BSM particles, yielding a SM-like $125 \mathrm{GeV}$ scalar. Thus the benchmark scenario chosen herein is of experimental interest.

What then can we conclude regarding the stability of the EW vacuum for this benchmark scenario? For all 200000 points generated complying with the choices above for the parameters and all constraints, we observe that the conditions for the possible existence of two minima, eqs. (3.7) and (3.8), are never satisfied. Thus, for this benchmark scenario, the EW vacuum is unique, and thus (at tree-level at least) entirely stable. Please beware:

\footnotetext{
${ }^{8}$ The reason for this is related to eqs. (2.11), which show it to be an efficient choice of parameters to fully specify the $2 \mathrm{HDM}$ potential.

${ }^{9}$ Notice how the exclusion bounds presented in figure 6 of [99] come from a 2HDM analysis which chooses values for the soft breaking such that $\lambda_{5}=0$. Though direct application of those exclusion bounds to our results is not possible, the model independent cross section limits presented in the afore-mentioned reference may exclude some of the parameter space discussed here.

${ }^{10}$ This implies that the coupling of $h$ to $\mathrm{Z}$ or $\mathrm{W}$ bosons and to fermions is very much SM-like.
} 


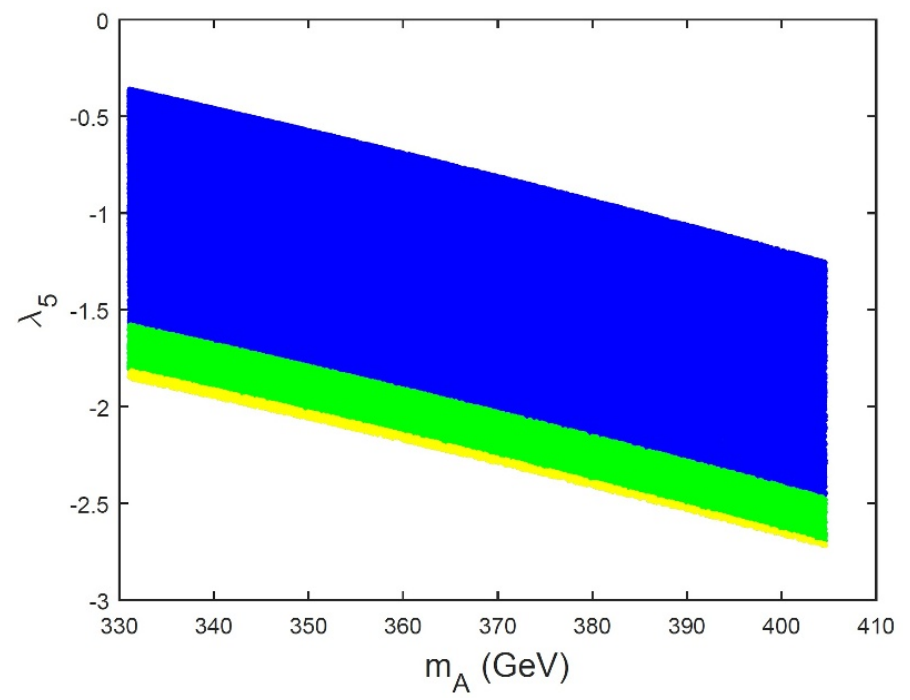

Figure 6. Scatter plots of $\lambda_{5}$ vs. $m_{A}$ for the low mass stable benchmark scenario considered. The colour code is the same as in figure 5 .

this does not mean that any choice of parameters in the decoupling regime will always fall into this category, although, as explained above for the type II model, large masses tend to yield stable EW vacua. Of course, there is no need to go into the decoupling regime to find parameters for which no non-degenerate vacua do not exist, the blue points in figure 5 show this to be true. Thus some regions of $2 \mathrm{HDM}$ parameter space have $\mathrm{EW}$ vacua which are unique at tree-level. Therefore, apart from the possibility of one-loop corrections to the potential originating deeper vacua as seems to be the case in the SM, the stability of the EW vacuum in such $2 \mathrm{HDM}$ parameter space regions is ensured and no tunneling calculations are needed.

Low mass stable scenario. Consider now a different choice of parameters corresponding to much lower masses for the extra scalars: $m_{H}=280 \mathrm{GeV}, m_{H^{ \pm}}=400 \mathrm{GeV}, \tan \beta=2.3$ and again $\sin (\beta-\alpha)=0.99$. This last choice all but ensures $h$ has SM-like behaviour. The value of $\tan \beta$ is chosen such as to comply with the exclusion in the $\tan \beta-m_{H^{ \pm}}$plane stemming from B-physics constraints [21]. And the low masses chosen for $H$ and $H^{ \pm}$are obviously interesting from the experimental point of view, as they raise the possibility of new particles discovered at LHC. As before, electroweak precision constraints force the pseudoscalar to be close in mass to the charged scalar, as can be appreciated from figure 6 . In this plot we show a "phase diagram" of the 2HDM parameter space. Unlike the plots in figure 5 - the colour code is the same here than in those plots - the parameter space now being scanned is truly a two-dimensional one, and thus figure 6 gives us a clearer picture of regions having different vacuum structure.

What we observe in figure 6 is the total absence of red points, and only a thin yellow strip where the EW vacuum could be the false vacuum. Indeed, for all points for which the EW vacuum is indeed a local minimum of the potential and not the global one, tunneling time calculations have revealed that the lifetime of the false vacuum is always far superior 

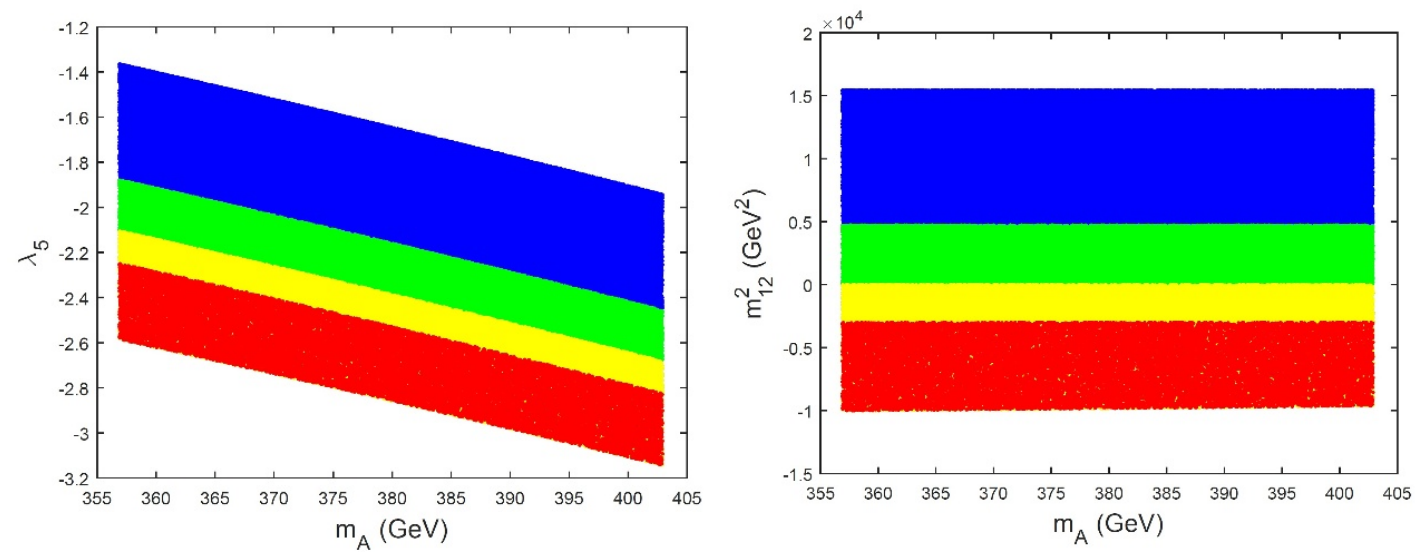

Figure 7. Scatter plots of $\lambda_{5}$ (left) and $m_{12}^{2}$ (right) vs. $m_{A}$ for the benchmark scenario considered in section 6.3. The colour code is the same as in figure 5 .

to the current age of the universe. Thus, even though for this benchmark scenario there may be dual minima, and "our" vacuum is not guaranteed to be the true vacuum of the model, it is nonetheless found to be either stable or incredibly long lived. Hence one must be careful to not exclude offhand regions of parameter space for which the discriminant $D$ from eq. (3.10) is negative $-D>0$ is a necessary and sufficient condition for the EW vacuum with $v=246 \mathrm{GeV}$ to be the global minimum of the theory, but as this example shows, points with $D<0$ may be entirely acceptable, having lifetimes larger than $T_{U}$. One must therefore be cautious in excluding regions of parameter space using the sign of the discriminant $D$, as was made in refs. [64, 100]: if $D<0$, tunneling times involving two-field bounce equations need to be computed, lest one is needlessly refusing phenomenologically acceptable combinations of $2 \mathrm{HDM}$ parameters.

\subsection{Second benchmark scenario: considerable exclusion}

The vacuum stability of the $2 \mathrm{HDM}$ may however change dramatically even for seemingly small variations in its parameters. Consider yet another choice of parameters, still corresponding to low masses for the extra scalars: $m_{H}=200 \mathrm{GeV}, m_{H^{ \pm}}=400 \mathrm{GeV}, \tan \beta=2.5$ and again $\sin (\beta-\alpha)=0.99$. Though this choice of parameters seems to be very similar to the previous benchmark considered, the allowed vacuum structure of the model is now quite different, as may be appreciated from figure 7. Notice how the region where two minima are in principle allowed (the yellow points) is now much larger, and how many red points now occur. In fact, for this region of parameter space, roughly $67 \%$ of all cases where "our" vacuum is the higher minimum yield a tunneling time inferior to the age of the universe. Globally, we find that for the points generated in this benchmark scenario which verify all theory and experimental constraints, roughly $11 \%$ have dangerous tunneling times. This is a much greater percentage than the one found for the blind parameter scan, showing that specific regions of the parameter space may be much more prone to dangerous false vacua than others. It is quite stunning how merely increasing by 0.2 the value of $\tan \beta$ and reducing by $80 \mathrm{GeV}$ the value of the heavier CP-even scalar may have such a drastic 
effect in the vacuum structure of the $2 \mathrm{HDM}$, but that simply reflects the complicated and non-obvious dependence on these parameters in eqs. (2.9), (3.7), (3.8) and (3.10). This not to mention the susceptibility of the tunneling time calculations to the geometry of the potential - which may be heavily influenced by changes in the potential's couplings - as illustrated in figures 2 and 3. The reader should also consider the importance of calculating the tunneling rates to the degenerate true vacua - as discussed in section 5, despite that degeneracy originating physically equivalent vacua, the lifetime of the false vacuum can change immensely if one does not take into account the existence of two possible true vacua it can decay into. In this present case, doing the lifetime calculation correctly taking into account both lower vacua yielded roughly $50 \%$ more dangerous red points than if we considered tunneling to only one of the lower vacua.

In the right of figure 7 we plot $m_{12}^{2}$ against $m_{A}$, illustrating nicely how all $\mathrm{EW}$ false vacua can only occur for negative values of the soft breaking parameter. This is a known feature of the $2 \mathrm{HDM}$ - negative discriminant $D$ seems to only occur for negative $m_{12}^{2}$, though there is no demonstration of this property. It would imply a correlation in the signs of the two last terms in the definition of the discriminant in eq. (3.10).

\section{Conclusions}

The $2 \mathrm{HDM}$ has a rich vacuum structure, with the possibility of coexisting electroweak breaking, CP conserving, non-degenerate vacua already at tree-level. Instability of a phenomenologically desirable vacuum, where both doublets have vevs $v_{1}$ and $v_{2}$ such that $v_{1}^{2}+v_{2}^{2}=(246 \mathrm{GeV})^{2}$ and elementary particles have their known masses, is therefore a possibility in the $2 \mathrm{HDM}$. The SM vacuum instabilities discussed in the literature are of a different nature - they occur due to radiative corrections to the potential, whereas in the 2HDM they may already arise at tree-level. Another difference in the 2HDM case involves the larger number of scalar fields which the potential possesses, which complicate considerably the calculation of the lifetime of eventual false vacua - clearly, a local minimum with a lifetime superior to the age of the universe should not, in principle, be excluded from consideration, since it might yield an acceptable description of known phenomenology.

In this work we analysed in depth the possibility of using the lifetime of false vacua as an exclusion tool of regions of $2 \mathrm{HDM}$ parameter space. The gauge freedom of the model allowed us to reduce the complexity of an a priori 8-field problem - and the physics of the models under discussion, coupled with the shape of the bounce equations describing the tunneling trajectories between vacua, permitted a further simplification. Vacua of different kinds cannot coexist in the $2 \mathrm{HDM}$ - existing theoretical results have proved that, for CP-conserving models, only two non-degenerate CP-conserving minima, both containing real vevs, can coexist. In models with explicit CP violation, on the other hand, the CP symmetry is not defined from the start, and minima with and without complex vevs can also coexist. We have shown that, in the CP-conserving $2 \mathrm{HDM}$ potential, the bounce equations, coupled with the necessary boundary conditions for the bounce, and the structure of the potential's derivatives dictated by CP symmetry, reduce the tunneling time calculation to a 2 -field problem. The remaining fields do not contribute, their bounce 
equations only allowing for trivial, vanishing solutions when the appropriate boundary conditions are taken into account. There are two crucial points in this reduction to a 2-field problem: (a) the bounce equations factorize on the fields whose vevs vanish at either minima; (b) at both the true and false vacua, only two fields have non-vanishing vevs. Both of these points are direct consequences of the physics involved, in this case the CP-conserving structure of the potential. On the other hand, for an explicitly CPviolating $2 \mathrm{HDM}$ potential, the factorization in the imaginary component field of one of the doublets no longer occurs, and minima with real and complex vevs can now coexist. Bounce solutions involving three fields become possible, something which is yet again a direct consequence of the physics involved, not simply a mathematical property.

Gauge freedom, the CP symmetry, the bounce equations and boundary conditions imposed by physics-limited vacuum structure of the $2 \mathrm{HDM}$ therefore allow us to reduce the computation of the tunneling time to a 2-field problem. However, the 2-field case produces a bizarre consequence. The $2 \mathrm{HDM}$ is invariant under a simultaneous sign redefinition for both scalar doublets, and no physical consequences should in principle arise from such a sign swap. Indeed, for any pair of solutions of the minimisation conditions of the potential, its symmetric is also a solution. This is a well-known, and trivial, property of 2HDM vacuum solutions. Any minimum found is in fact a "pair of minima", degenerate, separate in field space, each producing exactly the same physics. Hitherto the existence of such degenerate pairs of minima has been seen as nothing more than a curiosity, but here we have shown it may have significant impact in the lifetime of false vacua: indeed, the false vacuum can decay to a pair of degenerate true vacua, separated in field space, and the trajectory to each of the true vacua will not be, in general, the same. Hence the partial decay rates to each of the deeper vacua will in general be different, and the landscape of maxima and saddle points found along the trajectories to each true vacuum can indeed yield vastly different tunneling rates. We have found many instances where considering both tunneling possibilities yielded false vacua with lifetimes shorter than the age of the universe - whereas considering only one of the decaying possibilities seemed to indicate a stable false vacuum.

Applying the theoretical insights gained on generic scans of the 2HDM parameter space, we analysed which regions of that parameter space might be excluded on grounds of short EW vacuum lifetime. Generic scans over all allowed - under theory and experimental constraints - parameters show that the existence of non-degenerate minima is rare in the $2 \mathrm{HDM}$, and that even when a false vacuum occurs, its lifetime is often found to be superior to the age of the universe. The percentage of $2 \mathrm{HDM}$ parameter space points excluded in generic scans is then found to be of the order of a few percent. However, care must be exercised in reading this result, since the regions of $2 \mathrm{HDM}$ parameter space where non-degenerate minima occur are not uniformly distributed - and neither is the subset of those for which short-lived false vacua occur. We therefore proceeded to considering specific benchmark scenarios, illustrating how three very different regimes might occur. First we considered a choice for extra scalar masses and angles $\alpha$ and $\beta$ that pushed the theory well into the decoupling regime. Such a choice corresponds to a region of parameter space for which no non-degenerate minima exist in the potential, and as such the model is 
entirely stable at tree-level. The decoupling regime, of course, is not the only case where no non-degenerate vacua do not occur, the same does happen for smaller masses of the extra scalars. The second scenario considered studied a low-mass case for the extra scalars, and for which the possibility of a false EW vacuum now arises - certain regions of the considered parameter space had $D<0$, the discriminant which characterizes false vacua. However, for all such false vacua, the tunneling times towards the true vacuum were always found to be larger than $T_{U}$, and therefore stability is ensured. Thus the mere existence of a false vacuum should not be used per se to exclude regions of parameter space for which $D<0$ - tunneling times should and must be computed, and parameter exclusion should only be decided after that calculation.

Finally, we considered a low-mass scenario for which a large swath of parameter space is excluded on grounds of the short lifetime of the false vacuum found. The importance of a proper lifetime calculation - taking into account the existence of a pair of lower true vacua, related by sign changes in the values of the vevs - was emphasised. In fact, the number of dangerous vacua found can increase by as much as $50 \%$ when the full vacuum structure is taken into account. Though dangerous vacua are hard to pinpoint in terms of relations between potential couplings or physical observables, we observed that a negative discriminant only seems to occur for a negative soft breaking term $m_{12}^{2}$.

The overall conclusion of this work is that $2 \mathrm{HDM}$ vacuum instability at tree-level can have significant impact on parameter exclusion for certain regions of the parameter space - but that requires an appropriate calculation of the bounce solutions, taking into account the 2-field dynamics that CP-conservation allows us to study. Of crucial importance is also the seemingly trivial existence of pairs of degenerate, sign-swap-related, true vacua, since the lifetime of the false vacua may depend enormously on that fact. Generic blind scans of $2 \mathrm{HDM}$ parameters may suggest that the frequency of dangerous vacua is very small, but we have shown that they may be quite abundant for specific, experimentally-interesting, regions of parameter space. Though the current analysis was performed at tree-level, the significance of the results found is undeniable. Of course, from the existing SM studies, we can expect that radiative corrections will further complicate matters and bring more possibilities of vacuum instability. Thus a one-loop extension of the present work should be undertaken. It should also be considered, though, that unlike the SM, for which the top quark induces loop instabilities, in the 2HDM the larger scalar content of the model does in some cases counteract the fermion sector instability - it has been shown that the 2HDM, with a single tree-level vacuum, can be stable all the way up to the Planck scale, unlike what is claimed for the SM (see, for instance, [60, 64]). In which case, though higher order corrections to the present work are welcome, it may be that they do not bring major differences to the results shown here.

\section{Acknowledgments}

The work of VB and FC is carried out within the INFN project QFT-HEP. PF would like to thank the gratious hospitality and financial support from INFN, Sezione Catania, where this work was partially developed, as well as partial support under CERN fund 
grant CERN/FIS-PAR/0002/2017. This work is also partially supported by the Polish National Science Centre HARMONIA grant under contract UMO-2015/18/M/ST2/00518 (2016-2019).

Open Access. This article is distributed under the terms of the Creative Commons Attribution License (CC-BY 4.0), which permits any use, distribution and reproduction in any medium, provided the original author(s) and source are credited.

\section{References}

[1] ATLAS collaboration, Observation of a new particle in the search for the Standard Model Higgs boson with the ATLAS detector at the LHC, Phys. Lett. B 716 (2012) 1 [arXiv: 1207.7214] [INSPIRE].

[2] CMS collaboration, Observation of a new boson at a mass of $125 \mathrm{GeV}$ with the CMS experiment at the LHC, Phys. Lett. B 716 (2012) 30 [arXiv:1207.7235] [INSPIRE].

[3] ATLAS and CMS collaborations, Measurements of the Higgs boson production and decay rates and constraints on its couplings from a combined ATLAS and CMS analysis of the LHC pp collision data at $\sqrt{s}=7$ and 8 TeV, JHEP 08 (2016) 045 [arXiv:1606.02266] [INSPIRE].

[4] T.D. Lee, A Theory of Spontaneous T Violation, Phys. Rev. D 8 (1973) 1226 [InSPIRE].

[5] G.C. Branco, P.M. Ferreira, L. Lavoura, M.N. Rebelo, M. Sher and J.P. Silva, Theory and phenomenology of two-Higgs-doublet models, Phys. Rept. 516 (2012) 1 [arXiv:1106.0034] [INSPIRE].

[6] N.G. Deshpande and E. Ma, Pattern of Symmetry Breaking with Two Higgs Doublets, Phys. Rev. D 18 (1978) 2574 [INSPIRE].

[7] R. Barbieri, L.J. Hall and V.S. Rychkov, Improved naturalness with a heavy Higgs: An alternative road to LHC physics, Phys. Rev. D 74 (2006) 015007 [hep-ph/0603188] [INSPIRE].

[8] Q.-H. Cao, E. Ma and G. Rajasekaran, Observing the Dark Scalar Doublet and its Impact on the Standard-Model Higgs Boson at Colliders, Phys. Rev. D 76 (2007) 095011 [arXiv:0708.2939] [INSPIRE].

[9] L. Lopez Honorez, E. Nezri, J.F. Oliver and M.H.G. Tytgat, The Inert Doublet Model: An Archetype for Dark Matter, JCAP 02 (2007) 028 [hep-ph/0612275] [INSPIRE].

[10] P.M. Ferreira, R. Santos, M. Sher and J.P. Silva, Implications of the LHC two-photon signal for two-Higgs-doublet models, Phys. Rev. D 85 (2012) 077703 [arXiv:1112.3277] [INSPIRE].

[11] P.M. Ferreira, R. Santos, M. Sher and J.P. Silva, Could the LHC two-photon signal correspond to the heavier scalar in two-Higgs-doublet models?, Phys. Rev. D 85 (2012) 035020 [arXiv: 1201.0019] [INSPIRE].

[12] P. Bechtle, S. Heinemeyer, O. Stål, T. Stefaniak and G. Weiglein, HiggsSignals: Confronting arbitrary Higgs sectors with measurements at the Tevatron and the LHC, Eur. Phys. J. C 74 (2014) 2711 [arXiv:1305.1933] [INSPIRE].

[13] P. Bechtle, O. Brein, S. Heinemeyer, G. Weiglein and K.E. Williams, HiggsBounds: Confronting Arbitrary Higgs Sectors with Exclusion Bounds from LEP and the Tevatron, Comput. Phys. Commun. 181 (2010) 138 [arXiv:0811.4169] [INSPIRE]. 
[14] P. Bechtle, O. Brein, S. Heinemeyer, G. Weiglein and K.E. Williams, HiggsBounds 2.0.0: Confronting Neutral and Charged Higgs Sector Predictions with Exclusion Bounds from LEP and the Tevatron, Comput. Phys. Commun. 182 (2011) 2605 [arXiv:1102.1898] [INSPIRE].

[15] P. Bechtle et al., HiggsBounds-4: Improved Tests of Extended Higgs Sectors against Exclusion Bounds from LEP, the Tevatron and the LHC, Eur. Phys. J. C 74 (2014) 2693 [arXiv:1311.0055] [INSPIRE].

[16] O. Deschamps, S. Descotes-Genon, S. Monteil, V. Niess, S. T'Jampens and V. Tisserand, The Two Higgs Doublet of Type II facing flavour physics data, Phys. Rev. D 82 (2010) 073012 [arXiv: 0907.5135] [INSPIRE].

[17] F. Mahmoudi and O. Stal, Flavor constraints on the two-Higgs-doublet model with general Yukawa couplings, Phys. Rev. D 81 (2010) 035016 [arXiv:0907.1791] [INSPIRE].

[18] T. Hermann, M. Misiak and M. Steinhauser, $\bar{B} \rightarrow X_{s} \gamma$ in the Two Higgs Doublet Model up to Next-to-Next-to-Leading Order in QCD, JHEP 11 (2012) 036 [arXiv:1208.2788] [INSPIRE].

[19] M. Misiak et al., Updated NNLO QCD predictions for the weak radiative B-meson decays, Phys. Rev. Lett. 114 (2015) 221801 [arXiv:1503.01789] [INSPIRE].

[20] M. Misiak and M. Steinhauser, Weak radiative decays of the $B$ meson and bounds on $M_{H^{ \pm}}$ in the Two-Higgs-Doublet Model, Eur. Phys. J. C 77 (2017) 201 [arXiv:1702.04571] [INSPIRE].

[21] A. Arbey, F. Mahmoudi, O. Stal and T. Stefaniak, Status of the Charged Higgs Boson in Two Higgs Doublet Models, Eur. Phys. J. C 78 (2018) 182 [arXiv:1706.07414] [InSPIRE].

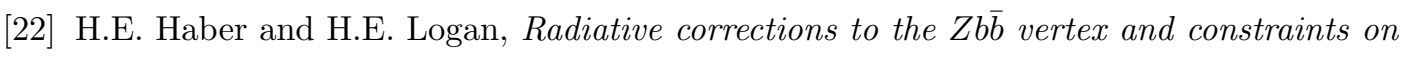
extended Higgs sectors, Phys. Rev. D 62 (2000) 015011 [hep-ph/9909335] [INSPIRE].

[23] P.M. Ferreira, R. Santos and A. Barroso, Stability of the tree-level vacuum in two Higgs doublet models against charge or CP spontaneous violation, Phys. Lett. B 603 (2004) 219 [Erratum ibid. B 629 (2005) 114] [hep-ph/0406231] [INSPIRE].

[24] A. Barroso, P.M. Ferreira and R. Santos, Charge and CP symmetry breaking in two Higgs doublet models, Phys. Lett. B 632 (2006) 684 [hep-ph/0507224] [INSPIRE].

[25] I.P. Ivanov, Minkowski space structure of the Higgs potential in 2HDM, Phys. Rev. D 75 (2007) 035001 [Erratum ibid. D 76 (2007) 039902] [hep-ph/0609018] [INSPIRE].

[26] I.P. Ivanov, Minkowski space structure of the Higgs potential in 2HDM. II. Minima, symmetries and topology, Phys. Rev. D 77 (2008) 015017 [arXiv:0710.3490] [INSPIRE].

[27] A. Barroso, P.M. Ferreira and R. Santos, Neutral minima in two-Higgs doublet models, Phys. Lett. B 652 (2007) 181 [hep-ph/0702098] [InSPIRE].

[28] A. Barroso, P.M. Ferreira, I.P. Ivanov, R. Santos and J.P. Silva, Evading death by vacuum, Eur. Phys. J. C 73 (2013) 2537 [arXiv:1211.6119] [InSPIRE].

[29] A. Barroso, P.M. Ferreira, I.P. Ivanov and R. Santos, Metastability bounds on the two Higgs doublet model, JHEP 06 (2013) 045 [arXiv: 1303.5098] [INSPIRE].

[30] ATLAS and CMS collaborations, Combined Measurement of the Higgs Boson Mass in pp Collisions at $\sqrt{s}=7$ and $8 \mathrm{TeV}$ with the ATLAS and CMS Experiments, Phys. Rev. Lett. 114 (2015) 191803 [arXiv: 1503.07589] [INSPIRE]. 
[31] ATLAS, CDF, CMS and D0 collaborations, First combination of Tevatron and LHC measurements of the top-quark mass, arXiv:1403.4427 [INSPIRE].

[32] S.R. Coleman, The Fate of the False Vacuum. 1. Semiclassical Theory, Phys. Rev. D 15 (1977) 2929 [Erratum ibid. D 16 (1977) 1248] [INSPIRE].

[33] C.G. Callan Jr. and S.R. Coleman, The Fate of the False Vacuum. 2. First Quantum Corrections, Phys. Rev. D 16 (1977) 1762 [inSPIRE].

[34] J. Elias-Miro, J.R. Espinosa, G.F. Giudice, G. Isidori, A. Riotto and A. Strumia, Higgs mass implications on the stability of the electroweak vacuum, Phys. Lett. B 709 (2012) 222 [arXiv: 1112.3022] [INSPIRE].

[35] G. Degrassi et al., Higgs mass and vacuum stability in the Standard Model at NNLO, JHEP 08 (2012) 098 [arXiv: 1205.6497] [INSPIRE].

[36] V. Branchina and E. Messina, Stability, Higgs Boson Mass and New Physics, Phys. Rev. Lett. 111 (2013) 241801 [arXiv: 1307.5193] [INSPIRE].

[37] V. Branchina, E. Messina and A. Platania, Top mass determination, Higgs inflation and vacuum stability, JHEP 09 (2014) 182 [arXiv:1407.4112] [INSPIRE].

[38] V. Branchina, E. Messina and M. Sher, Lifetime of the electroweak vacuum and sensitivity to Planck scale physics, Phys. Rev. D 91 (2015) 013003 [arXiv:1408.5302] [InSPIRE].

[39] V. Branchina, E. Messina and D. Zappala, Impact of Gravity on Vacuum Stability, EPL 116 (2016) 21001 [arXiv: 1601.06963] [INSPIRE].

[40] V. Branchina and E. Messina, Stability and UV completion of the Standard Model, EPL 117 (2017) 61002 [arXiv:1507.08812] [INSPIRE].

[41] E. Bentivegna, V. Branchina, F. Contino and D. Zappalà, Impact of New Physics on the $E W$ vacuum stability in a curved spacetime background, JHEP 12 (2017) 100 [arXiv: 1708.01138] [INSPIRE].

[42] A. Masoumi, K.D. Olum and B. Shlaer, Efficient numerical solution to vacuum decay with many fields, JCAP 01 (2017) 051 [arXiv:1610.06594] [INSPIRE].

[43] J.F. Gunion and H.E. Haber, The CP conserving two Higgs doublet model: The approach to the decoupling limit, Phys. Rev. D 67 (2003) 075019 [hep-ph/0207010] [INSPIRE].

[44] A. Pich and P. Tuzon, Yukawa Alignment in the Two-Higgs-Doublet Model, Phys. Rev. D 80 (2009) 091702 [arXiv:0908.1554] [INSPIRE].

[45] M. Jung, A. Pich and P. Tuzon, Charged-Higgs phenomenology in the Aligned two-Higgs-doublet model, JHEP 11 (2010) 003 [arXiv:1006.0470] [INSPIRE].

[46] M. Jung, A. Pich and P. Tuzon, The $B \rightarrow X_{s} \gamma$ Rate and CP Asymmetry within the Aligned Two-Higgs-Doublet Model, Phys. Rev. D 83 (2011) 074011 [arXiv:1011.5154] [INSPIRE].

[47] P.M. Ferreira, L. Lavoura and J.P. Silva, Renormalization-group constraints on Yukawa alignment in multi-Higgs-doublet models, Phys. Lett. B 688 (2010) 341 [arXiv:1001.2561] [INSPIRE].

[48] S. Gori, H.E. Haber and E. Santos, High scale flavor alignment in two-Higgs doublet models and its phenomenology, JHEP 06 (2017) 110 [arXiv:1703.05873] [INSPIRE].

[49] S.L. Glashow and S. Weinberg, Natural Conservation Laws for Neutral Currents, Phys. Rev. D 15 (1977) 1958 [INSPIRE]. 
[50] E.A. Paschos, Diagonal Neutral Currents, Phys. Rev. D 15 (1977) 1966 [inSPIRE].

[51] G. Kreyerhoff and R. Rodenberg, Renormalization Group Analysis of Coleman-Weinberg Symmetry Breaking in Two Higgs Models, Phys. Lett. B 226 (1989) 323 [InSPIRE].

[52] S. Nie and M. Sher, Vacuum stability bounds in the two Higgs doublet model, Phys. Lett. B 449 (1999) 89 [hep-ph/9811234] [INSPIRE].

[53] S. Kanemura, T. Kasai and Y. Okada, Mass bounds of the lightest CP even Higgs boson in the two Higgs doublet model, Phys. Lett. B 471 (1999) 182 [hep-ph/9903289] [INSPIRE].

[54] P.M. Ferreira and D.R.T. Jones, Bounds on scalar masses in two Higgs doublet models, JHEP 08 (2009) 069 [arXiv:0903.2856] [INSPIRE].

[55] F. Bezrukov, M. Yu. Kalmykov, B.A. Kniehl and M. Shaposhnikov, Higgs Boson Mass and New Physics, JHEP 10 (2012) 140 [arXiv:1205.2893] [INSPIRE].

[56] D. Buttazzo et al., Investigating the near-criticality of the Higgs boson, JHEP 12 (2013) 089 [arXiv: 1307.3536] [INSPIRE].

[57] N. Chakrabarty, U.K. Dey and B. Mukhopadhyaya, High-scale validity of a two-Higgs doublet scenario: a study including LHC data, JHEP 12 (2014) 166 [arXiv:1407.2145] [INSPIRE].

[58] D. Das and I. Saha, Search for a stable alignment limit in two-Higgs-doublet models, Phys. Rev. D 91 (2015) 095024 [arXiv: 1503.02135] [INSPIRE].

[59] D. Chowdhury and O. Eberhardt, Global fits of the two-loop renormalized Two-Higgs-Doublet model with soft $Z_{2}$ breaking, JHEP 11 (2015) 052 [arXiv:1503.08216] [INSPIRE].

[60] P. Ferreira, H.E. Haber and E. Santos, Preserving the validity of the Two-Higgs Doublet Model up to the Planck scale, Phys. Rev. D 92 (2015) 033003 [Erratum ibid. D 94 (2016) 059903] [arXiv: 1505.04001] [INSPIRE].

[61] N. Chakrabarty and B. Mukhopadhyaya, High-scale validity of a two Higgs doublet scenario: metastability included, Eur. Phys. J. C 77 (2017) 153 [arXiv:1603.05883] [INSPIRE].

[62] V. Cacchio, D. Chowdhury, O. Eberhardt and C.W. Murphy, Next-to-leading order unitarity fits in Two-Higgs-Doublet models with soft $\mathbb{Z}_{2}$ breaking, JHEP 11 (2016) 026 [arXiv: 1609.01290] [INSPIRE].

[63] N. Chakrabarty and B. Mukhopadhyaya, High-scale validity of a two Higgs doublet scenario: predicting collider signals, Phys. Rev. D 96 (2017) 035028 [arXiv: 1702.08268] [INSPIRE].

[64] P. Basler, P.M. Ferreira, M. Mühlleitner and R. Santos, High scale impact in alignment and decoupling in two-Higgs doublet models, Phys. Rev. D 97 (2018) 095024 [arXiv: 1710.10410] [INSPIRE].

[65] S. Kanemura, T. Kubota and E. Takasugi, Lee-Quigg-Thacker bounds for Higgs boson masses in a two doublet model, Phys. Lett. B 313 (1993) 155 [hep-ph/9303263] [INSPIRE].

[66] A.G. Akeroyd, A. Arhrib and E.-M. Naimi, Note on tree level unitarity in the general two Higgs doublet model, Phys. Lett. B 490 (2000) 119 [hep-ph/0006035] [INSPIRE].

[67] J. Horejsi and M. Kladiva, Tree-unitarity bounds for THDM Higgs masses revisited, Eur. Phys. J. C 46 (2006) 81 [hep-ph/0510154] [INSPIRE]. 
[68] B. Grinstein, C.W. Murphy and P. Uttayarat, One-loop corrections to the perturbative unitarity bounds in the CP-conserving two-Higgs doublet model with a softly broken $\mathbb{Z}_{2}$ symmetry, JHEP 06 (2016) 070 [arXiv:1512.04567] [INSPIRE].

[69] M.E. Peskin and T. Takeuchi, A new constraint on a strongly interacting Higgs sector, Phys. Rev. Lett. 65 (1990) 964 [INSPIRE].

[70] M.E. Peskin and T. Takeuchi, Estimation of oblique electroweak corrections, Phys. Rev. D 46 (1992) 381 [INSPIRE].

[71] I. Maksymyk, C.P. Burgess and D. London, Beyond S, T and U, Phys. Rev. D 50 (1994) 529 [hep-ph/9306267] [INSPIRE].

[72] GFitter Group collaboration, M. Baak et al., The global electroweak fit at NNLO and prospects for the LHC and ILC, Eur. Phys. J. C 74 (2014) 3046 [arXiv:1407.3792] [INSPIRE].

[73] LEP, DELPHI, OPAL, ALEPH and L3 collaborations, G. Abbiendi et al., Search for Charged Higgs bosons: Combined Results Using LEP Data, Eur. Phys. J. C 73 (2013) 2463 [arXiv:1301.6065] [INSPIRE].

[74] R.V. Harlander, S. Liebler and H. Mantler, SusHi: A program for the calculation of Higgs production in gluon fusion and bottom-quark annihilation in the Standard Model and the MSSM, Comput. Phys. Commun. 184 (2013) 1605 [arXiv:1212.3249] [INSPIRE].

[75] R.V. Harlander, S. Liebler and H. Mantler, SusHi Bento: Beyond NNLO and the heavy-top limit, Comput. Phys. Commun. 212 (2017) 239 [arXiv:1605.03190] [INSPIRE].

[76] L. Wang, F. Zhang and X.-F. Han, Two-Higgs-doublet model of type-II confronted with the LHC run-I and run-II data, Phys. Rev. D 95 (2017) 115014 [arXiv:1701.02678] [INSPIRE].

[77] P.M. Ferreira, S. Liebler and J. Wittbrodt, $p p \rightarrow A \rightarrow Z h$ and the wrong-sign limit of the two-Higgs-doublet model, Phys. Rev. D 97 (2018) 055008 [arXiv:1711.00024] [InSPIRE].

[78] P.M. Ferreira, J.F. Gunion, H.E. Haber and R. Santos, Probing wrong-sign Yukawa couplings at the LHC and a future linear collider, Phys. Rev. D 89 (2014) 115003 [arXiv: 1403.4736] [INSPIRE].

[79] P.M. Ferreira, R. Guedes, M.O.P. Sampaio and R. Santos, Wrong sign and symmetric limits and non-decoupling in 2HDMs, JHEP 12 (2014) 067 [arXiv:1409.6723] [INSPIRE].

[80] B. Dumont, J.F. Gunion, Y. Jiang and S. Kraml, Constraints on and future prospects for Two-Higgs-Doublet Models in light of the LHC Higgs signal, Phys. Rev. D 90 (2014) 035021 [arXiv: 1405.3584] [INSPIRE].

[81] D. Fontes, J.C. Romão and J.P. Silva, A reappraisal of the wrong-sign hbb coupling and the study of $h \rightarrow Z \gamma$, Phys. Rev. D 90 (2014) 015021 [arXiv:1406.6080] [INSPIRE].

[82] J. Bernon, J.F. Gunion, Y. Jiang and S. Kraml, Light Higgs bosons in Two-Higgs-Doublet Models, Phys. Rev. D 91 (2015) 075019 [arXiv:1412.3385] [INSPIRE].

[83] A. Biswas and A. Lahiri, Alignment, reverse alignment and wrong sign Yukawa couplings in two Higgs doublet models, Phys. Rev. D 93 (2016) 115017 [arXiv:1511.07159] [INSPIRE].

[84] T. Modak, J.C. Romão, S. Sadhukhan, J.P. Silva and R. Srivastava, Constraining wrong-sign hbb couplings with $h \rightarrow \Upsilon \gamma$, Phys. Rev. D 94 (2016) 075017 [arXiv: 1607.07876] [INSPIRE]. 
[85] G.V. Dunne, Functional determinants in quantum field theory, J. Phys. A 41 (2008) 304006 [arXiv:0711.1178] [INSPIRE].

[86] G.V. Dunne and H. Min, Beyond the thin-wall approximation: Precise numerical computation of prefactors in false vacuum decay, Phys. Rev. D 72 (2005) 125004 [hep-th/0511156] [INSPIRE].

[87] P.B. Arnold and S. Vokos, Instability of hot electroweak theory: bounds on $m_{H}$ and $M_{t}$, Phys. Rev. D 44 (1991) 3620 [InSPIRE].

[88] A. Pilaftsis and C.E.M. Wagner, Higgs bosons in the minimal supersymmetric standard model with explicit CP-violation, Nucl. Phys. B 553 (1999) 3 [hep-ph/9902371] [inSPIRE].

[89] I.F. Ginzburg, M. Krawczyk and P. Osland, Two Higgs doublet models with CP violation, in Linear colliders. Proceedings, International Workshop on physics and experiments with future electron-positron linear colliders, LCWS 2002, Seogwipo, Jeju Island, Korea, August 26-30, 2002, pp. 703, hep-ph/0211371 CERN-TH-2002-330 (2002).

[90] W. Khater and P. Osland, CP violation in top quark production at the LHC and two Higgs doublet models, Nucl. Phys. B 661 (2003) 209 [hep-ph/0302004] [INSPIRE].

[91] A.W. El Kaffas, P. Osland and O.M. Ogreid, CP violation, stability and unitarity of the two Higgs doublet model, Nonlin. Phenom. Complex Syst. 10 (2007) 347 [hep-ph/0702097] [INSPIRE].

[92] A.W. El Kaffas, W. Khater, O.M. Ogreid and P. Osland, Consistency of the two Higgs doublet model and CP-violation in top production at the LHC, Nucl. Phys. B 775 (2007) 45 [hep-ph/0605142] [INSPIRE].

[93] A. Wahab El Kaffas, P. Osland and O.M. Ogreid, Constraining the Two-Higgs-Doublet-Model parameter space, Phys. Rev. D 76 (2007) 095001 [arXiv:0706.2997] [INSPIRE].

[94] P. Osland, P.N. Pandita and L. Selbuz, Trilinear Higgs couplings in the two Higgs doublet model with CP-violation, Phys. Rev. D 78 (2008) 015003 [arXiv:0802.0060] [INSPIRE].

[95] B. Grzadkowski and P. Osland, Tempered Two-Higgs-Doublet Model, Phys. Rev. D 82 (2010) 125026 [arXiv:0910.4068] [INSPIRE].

[96] A. Arhrib, E. Christova, H. Eberl and E. Ginina, CP violation in charged Higgs production and decays in the Complex Two Higgs Doublet Model, JHEP 04 (2011) 089 [arXiv: 1011.6560] [INSPIRE].

[97] A. Barroso, P.M. Ferreira, R. Santos and J.P. Silva, Probing the scalar-pseudoscalar mixing in the $125 \mathrm{GeV}$ Higgs particle with current data, Phys. Rev. D 86 (2012) 015022 [arXiv: 1205.4247] [INSPIRE].

[98] X.-J. Xu, Tree-level vacuum stability of two-Higgs-doublet models and new constraints on the scalar potential, Phys. Rev. D 95 (2017) 115019 [arXiv:1705.08965] [INSPIRE].

[99] ATLAS collaboration, Search for a heavy Higgs boson decaying into a $Z$ boson and another heavy Higgs boson in the lebb final state in pp collisions at $\sqrt{s}=13 \mathrm{TeV}$ with the ATLAS detector, Phys. Lett. B 783 (2018) 392 [arXiv:1804.01126] [InSPIRE].

[100] F. Staub, Reopen parameter regions in Two-Higgs Doublet Models, Phys. Lett. B 776 (2018) 407 [arXiv: 1705. 03677] [inSPIRE]. 